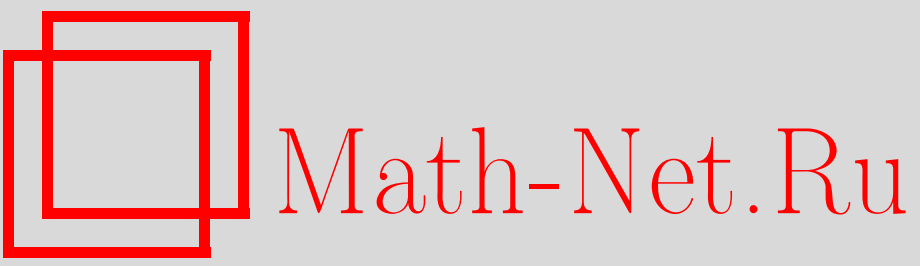

В. И. Арнольд, Топологические проблемы теории распространения волн, УМН, 1996, том 51, выпуск 1, 3-50

DOI: https://doi.org/10.4213/rm919

Использование Общероссийского математического портала Math-Net.Ru подразумевает, что вы прочитали и согласны с пользовательским соглашением

http://www.mathnet.ru/rus/agreement

Параметры загрузки:

IP : 54.224 .187 .69

26 апреля 2023 г., 13:18:57 


\title{
ТОПОЛОГИЧЕСКИЕ ПРОБЛЕМЫ ТЕОРИИ РАСПРОСТРАНЕНИЯ ВОЛН
}

\author{
В. И. АРнольд \\ СОДЕРЖАНИЕ
}

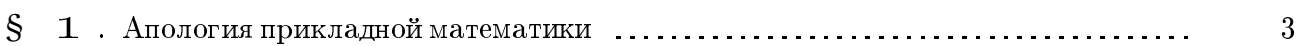

$\S 2$. Теоремы о четырех точках возврата ............................ 7

$\S 3$. Теорема Гурвица-Келлога-Табачникова типа Штурма . . . . . . . . . . . . . . 14

$\S \quad 4$. Тригонометрические аппроксимации ............................. 20

$\S 5$. Лагранжевы пересечения в симплектической топологии $\ldots \ldots \ldots \ldots \ldots \ldots \ldots . .22$

$\S 6$. Лежандровы зацепления в контактной топологии $\ldots \ldots \ldots \ldots \ldots \ldots \ldots \ldots \ldots . . \ldots \ldots$

$\S 7$. Лагранжев коллапс и точки возврата каустик . . . . . . . . . . . . . . . . . 28

$\S 8$. Лежандров коллапс и точки возврата фронтов . . . . . . . . . . . . . . . . . . . . 31

$\S 9$. Пространственные кривые и их точки уплощения $\ldots \ldots \ldots \ldots \ldots \ldots \ldots \ldots \ldots \ldots . . . \ldots \ldots \ldots$

$\S 1$ О. Вершины выпуклых пространственных кривых .................... 36

$\S 11$. Приложения в теории экстатических точек плоских кривых $\ldots \ldots \ldots \ldots \ldots . .41$

$\S 12$. Многомерные обобщения теории Штурма . ......................... 42

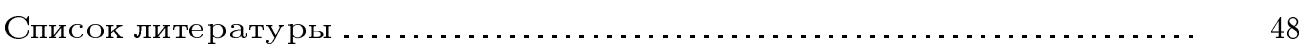

\section{§1. Апология прикладной математики}

Обычное (хотя обычно и скрываемое) мнение как чистых математиков, так и теоретических физиков об "индустриальной и прикладной" математике состоит в том, что это - мафия слабых мыслителей, неспособных произвести никакие важные научные результаты, а просто эксплуатирующих достижения чистых математиков прошлых поколений, что члены этой мафии более заинтересованы в деньгах, чем в науке и безнадежно этим испорчены.

“Они так скромны, - сказал однажды один чистьй математик, - что не надеются добиться чего-либо прямым честньм путем; им приходится отделяться от математиков просто для того, чтобы избежать честного соревнования".

\footnotetext{
Доклад на открытии конгресса индустриальной и прикладной математики в Гамбурге, 3 июля 1995 г.
}

(C) В.и. Арнольд 1996 
Я не думаю, чтобы эта характеристика прикладных математиков была полностью заслуженной. Достижения склонного к бизнесу Галилея вызывают не меньшее восхишение, чем результаты чистого философа Паскаля.

Разница между чистой и прикладной математикой не научная, а лишь социальная. Чистому математику платят за то, чтобы он открывал новые математические факты. Прикладному математику платят за решение вполне определенных задач.

ПримеР. Колумб вначале действовал подобно прикладному ученому, стараясь найти путь в Индию, за что ему и платили.

В конце его путешествие напоминало деятельность чистого математика. Заметим, что непосредственная, немедленная польза для испанской экономики от открытий Колумба была гораздо меньшей, чем от каботажных плаваний рядовых капитанов.

Маяковский хорошо описал разницу между чистой и прикладной математикой в "Как делать стихи": “Человек, открьвший, что дважды два четыре, был великим математиком, даже если он открыл это, считая окурки. Те, кто теперь считают по той же формуле гораздо большие предметы, например - паровозы, вообше не математики".

Теория алгебраических кривых над конечными полями сейчас стала прикладной математикой, оплачиваемой ЦРУ, КГБ и другими подобными организациями. Проблема Ферма тоже была бы прикладной, если бы за ее решение платили. Опасность разделения математики на части хорошо осознавали многие математики двадшатого века. Герман Вейль писал: "В наше время за душу каждой области математики борются ангел топологии и дьявол абстрактной алгебры" [1].

В первой половине века дљявол побеждал. Вслед за Лагранжем, изгнавшим из математики чертежи, пришли алгебраисты и аксиоматизаторы - сперва Гильберт, затем Бурбаки.

ПРИмЕР. Определим произведение натуральных чисел при помощи алгоритма умножения "столбиком". Тогда коммутативность умножения станет трудно доказьваемой теоремой. Формальное доказательство этой теоремы можно затем заставить учить школьников (или студентов), терроризируя их и поднимая на небывалую высоту авторитет как учителей, так и самой их науки.

Поколения математиков были обучены этим методом, не имея никаких контактов ни с какой другой математикой. В результате они не способны понимать никакие другие науки и с увлечением занимаются скучными деталями малоинтересных обобшений достижений своих учителей.

Гильберт провозгласил демократический принцип, по которому всякая система аксиом имеет равное право быть исследованной, а значение математического достижения определяется лишь его трудностью, как в альпинизме. Результатом явился развод "чистой" математики со всеми науками, преступная по отношению к обучаемьм система обучения математике и образ математики в общественном мнении как опасной паразитической секты на теле науки и техники, состоящей из жрецов умирающей религии, вроде друидов.

Ландау говорил: “Зачем это математики складьвают простые числа? Простые числа созданы, чтобы их умножать" (теорема о представимости всякого достаточно большого нечетного числа в виде суммы трех простых считается одним из главных достижений математики). 
Мстя за унижения, перенесенные в школе, правители большинства стран, подобно свиньям под дубом, предпринимают теперь, после уменьшения военного противостояния, усилия для изничтожения математики, особенно “чистой”. Правительство США недавно выяснило, что $85 \%$ имеющихся и обучающихся математиков стране не нужны. Без звездных войн не нужны ни суперколлайдеры, ни математики. Обсуждаются различные проекты, как сократить число математиков в семь раз. Американские специалисты считают, что на это потребуется лет десять.

К сожалению, нельзя не признать, что “чистые” математики своими руками сделали все для того, чтобы создать описанное общественное мнение. Аксиоматическидедуктивньй метод, приведший к изгнанию всех примеров (а особенно мотивировок вводимых определений) в преподавании математики на всех уровнях прежде всего ответственен за это.

Р.Фейнман [2] ярко описывает этот метод преподавания ("приведения в состояние самораспространяюшейся псевдообразованности") в книге "Вы конечно шутите, мистер Фейнман”, рассматривая преподавание физики в Бразилии в первые послевоенные годы. Читая Фейнмана, я не раз краснел - настолько этот “бразильский" метод преподавания физики похож на наше математическое преподавание.

Фейнман приводит такой пример. Лектор говорит, что моментом инерции материальной точки относительно оси называется произведение массы на квадрат расстояния до оси. Студенты записывают определение. Кажется, здесь все в порядке. Но Фейнман объясняет, что такое преподавание совершенно недопустимо.

Нужно объяснить, что груз, закрепленный на двери вблизи ее оси, почти не чувствуется при открывании и закрывании двери, а закрепленньй у ручки - сильно мешает. Без подобного примера определение бессмысленно - оно годится только для того, чтобы на экзамене ответить на вопрос: “сформулируйте определение момента инерции”. Примеры, показываюшие беспомошность так обученных студентов, желаюшие могут найти в книге Фейнмана.

Вот несколько примеров из моего педагогического опыта в Париже. Во время письменного экзамена студент четвертого курса спросил меня: "Я забыл калькулятор подскажите, пожалуйста, 4/7 больше единицы или меньше?”. Четыре седьмых было тем самьм числом, от которого зависела сходимость интеграла, управляющего поведением исследуемой динамической системы. Это был хороший студент, но вот простые дроби он, видимо, учил по французскому варианту “бразильского” метода!

Студенты высшей нормальной школы в Париже спросили меня: "Почему Вы называете кольцо формальных степенных рядов локальным? Разве оно удовлетворяет аксиомам локального кольца?" Для неспециалистов поясню, что заданньй вопрос аналогичен вопросу “почему Вы называете окружность коническим сечением?” Это были лучшие студенты - математики Франции. По-видимому, какой-то преступњй алгебраист обучил их аксиомам колец (и даже локальных колец), не приводя ни одного примера (и, в частности, не объяснив происхождение термина “локальное”).

Из-за ошибки в начальном условии фазовая кривая гамильтоновой системы на плоскости оказалась не сепаратрисой седла, а замкнутой кривой. Это привело к следующему решению задачи о пределе решения при $t$, стремяшемся к бесконечности: "по теореме 45 - на письменном экзамене разрешается пользоваться любьми источниками - сушествует $T>0$ такое, что значение $\varphi(T)$ решения в момент $T$ равно начальному значению". Далее по индукции совершенно строго доказьвается (с использованием 
теоремы единственности), что $\varphi(n T)=\varphi(0)$ при любом целом $n$.

Из всего этого делается безукоризненньй вьвод: “предел равен начальному значению”! С точки зрения дедуктивно-аксиоматической математики во всех этих рассуждениях нет ни одной ошибки: ошибочна лишь формулировка задачи.

Ясно, однако, что автор ничего не понимает, а умеет лишь доказывать. Бессмысленность и даже преступность системы обучения, доводящей явно неглупых людей до такого состояния, представляется мне очевидной. Для “прикладной” деятельности такие “знания" бесполезны и даже опасны (последствия могут ведь носить характер Чернобыльской аварии).

Целью математической лекции должен быть не логический вьвод одних непонятных утверждений из других (столь же непонятных): слушателям нужно объяснить, о чем идет речь, и научить их пользоваться не только изложенными результатами, но - и это главное - также методами и идеями.

Вслед за преврашением математических лекций в подобие богослужений, не предполагающих какого-либо их понимания слушателями, в богослужения стали превращаться и математические конференции и конгрессы. Разница между мессой и докладом на Конгрессе (например, нынешнем) состоит, однако, в том, что за вход на доклад слушатели должны платить, и немалую сумму. Это, впрочем, неудивительно - ведь за каждую ночь, проведенную докладчиком в гостинице, где происходит этот Конгресс, его организаторы платят отелю сумму, соответствующую моей московской заработной плате за несколько месящев . . . .

В России развод между “чистой” и “прикладной” математикой никогда не был полным, и почти каждый математик сочетал и то, и другое. Например, все мои работы по динамическим системам (включая работы по небесной механике, магнитным ловушкам, сердечным аритмиям и гидродинамической устойчивости) выполнены в рамках “чистой” математики в МГУ, а работы по алгебраической геометрии, теории особенностей, группам Кокстера и теории кос - прикладные (выполнены для Московского института электронного машиностроения в связи с исследованием теплового режима в кристаллической среде).

Оставшаяся часть этого доклада посвяшена результатам о топологически необходимых особенностях и бифуркациях в теории распространения волн.

Я выбрал этот пример взаимодействия между “чистой” и “прикладной” математикой потому, что здесь проблемы из прикладной области (теории волновых фронтов, каустик и лучей в оптике, механике и в теории оптимального управления и игр) привели недавно к открытию множества новых фактов как в чистой математике, так и в приложениях, связьвая меж ду собой

- топологические свойства сферических функций (см. §12);

- теорию Морса критических точек (см. $\S 5,6)$;

- теорию осциллящий Штурма (см. $\S 3,4,12)$;

- теорию чебышевских систем (см. $\S \S 9,10)$;

- топологию плоских кривых и узлов (см. [9], [10], [20]-[24]);

- дифференциальную и алгебраическую геометрию (см. [12], [14], [64]);

- квантовую теорию поля (см. [22], [24]);

- статистическую физику (см. [17]);

с одной стороны, 
и симплектическую и контактную геометрию лагранжевых и лежандровых многообразий и отображений - с другой (см. [5], [8], [11], [27], [30]).

Я сформулирую также ряд топологических и алгебро-геометрических гипотез, связанных с теорией волн и теорией сферических функций.

\section{§ 2. Теоремы о четырех точках возврата}

Начну с нескольких теорем, общим для которых является появление числа "четыре" в ответе. На первый взгляд не видно, что между ними обшего. Но в действительности все эти факты - проявления одного и того же топологического свойства лагранжевых и лежандровых особенностей в симплектической и контактной топологии фазового пространства механики и контактной структуры оптики и теории управления.

ПримеР 1. Последняя геометрическая теорема Якоби. Рассмотрим выпуклую поверхность (например, поверхность эллипсоида) и точку на ней. Выпустим из этой точки геодезическую по некоторому направлению.

ОПРЕДЕЛЕНИЕ. Сопряженной точкой (данной начальной точки) называется точка пересечения данной геодезической с бесконечно-близкой к ней геодезической, выходящей из данной начальной точки (рис. 1).

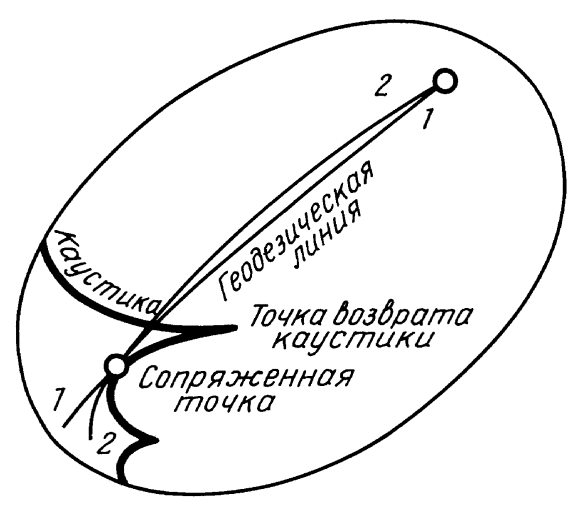

Рис. 1. Каустика точки на вьпуклой поверхности

Первой сопряженной точкой северного полюса сферы вдоль любой геодезической является южньй полюс. Для поверхности обшего положения (например, для эллипсоида) сопряженные точки вдоль разных геодезических различны.

ОПРЕДЕЛЕНИЕ. Каустикой исходной точки назьвается кривая, образованная ее сопряженными точками (вдоль всех выходящих из исходной точки геодезических).

Якоби (в своих “Лекциях по динамике" [3]) заметил, что каустика не может быть гладкой кривой. Каустика точки обшего положения на поверхности обшего положения обязательно имеет точки возврата. (В окрестности такой точки кривая диффеоморфна полукубической параболе, $y^{2}=x^{3}$.) 
Эта теорема Якоби, доказанная им при помощи топологических соображений, явилась, по-видимому, исторически первым результатом "вариационного исчисления в целом".

Из топологических соображений ясно, что число точек возврата на каустике четно. Доказав это, Якоби замечает, что в случае эллипсоида это четное число всегда равно четырем.

Лекции Якоби опубликованы посмертно. Последняя статья в последнем томе собрания сочинений Якоби - неоконченное сочинение о точках возврата каустик. Оно сопровождается неуспешными (и довольно наивными) попытками учеников Якоби восстановить его рассуждения.

Насколько мне известно, утверждение Якоби о четырех точках возврата каустики на эллипсоиде так никогда и не было доказано.

Якоби проинтегрировал уравнение геодезических на эллипсоиде (в тета-функциях от двух переменных). Таким образом, вопрос о числе точек возврата каустики, в сущности, относится к алгебраической геометрии.

Однако современная алгебраическая геометрия, с ее уклоном в теорию чисел и с потерей интереса к реальным задачам, по-видимому, не в состоянии справиться с задачей Якоби. Верно ли утверждение Якоби о том, что каустики эллипсоидов имеют четыре точки возврата, не известно и сегодня. Не исключено даже, что численное интегрирование уравнений геодезических обнаружит контрпример (хотя это противоречило бы общему принципу минимальности топологических инвариантов алгебраических объектов).

Положение дел с четырьмя точками возврата каустик выпуклых поверхностей на сегодня следуюшее. Заметим прежде всего, что каустика имеет много компонент: первые сопряженные точки образуют первую каустику, вторые - вторую и т. д. Например, первая каустика северного полюса сферы - южньй полюс, вторая - северньй, третья - южньй и т. д.

1) Первая каустика имеет не менее четырех точек возврата. Этот классический результат вариационного исчисления в целом вытекает из анализа топологии страта Максвелла - множества тех точек поверхности, которые соединены с исходной точкой более чем одной минимальной геодезической.

Для точки и поверхности обшего положения страт Максвелла представляет собой дерево. Концевые точки этого дерева являются точками возврата каустики.

Всякое дерево имеет не меньше двух конщевых точек. Из этого и следует сушествование не менее четырех точек возврата первой каустики. А именно, рассмотрим расстояние вдоль геодезической от исходной точки до первой сопряженной точки как функцию на окружности выходящих из исходной точки направлений. Концевые точки дерева являются точками локального минимума этой функции на окружности. Она имеет не менее двух точек локального максимума. Эти точки тоже соответствуют точкам возврата первой каустики (они уже не являются концевыми точками страта Максвелла и, вообще говоря, вовсе ему не принадлежат).

Минимумы и максимумы нашей функции на окружности вместе и дают не менее четырех точек возврата первой каустики.

Недавно Закалюкин [4] исследовал топологию страта Максвелла для следующих каустик, но результаты здесь менее полны. 
2) Число точек возврата на $n$-й каустике не меньше четырех, если поверхность достаточно близка к сфере [5]. Близость здесь, однако, повышается с ростом $n$, так что для каждой конкретной поверхности получается лишш утверждение о конечном числе каустик.

ГИПотЕЗА. Каждая каустика общего положения на каждой выпуклой поверхности положительной кривизны имеет не менее четырех точек возврата.

Таким образом, гипотетически малость возмущения сферы не нужна. Однако даже для поверхностей, сколь угодно близких к сфере, не доказано присутствие не менее четырех точек возврата на каустиках всех порядков одновременно.

Вычисления по теории возмушений сводят задачу о точках возврата каустик на поверхностях, близких к сфере, к теореме Гурвица типа Штурма о рядах Фурье (см. §3 ниже). Доказательство в [5] переносит этот результат с бесконечно-малых возмущений сферы на конечные.

Пример 2. Теорема о четырех вершинах. Вершиной плоской кривой называется критическая точка кривизны. Классическая теорема о четырех вершинах [6] утверждает, что выпуклая замкнутая кривая на плоскости имеет не менее четырех вериин.

Например, для эллипса вершинами являются концы большой и малой осей.

На первый взгляд кажется, что теорема о четырех вершинах относится к римановой геометрии плоскости, но это не так. Стоит немного изменить риманову метрику плоскости, как результат станет неверным. Геодезическая кривизна подходящей кривой, сколь угодно близкой к окружности, на подходящей поверхности, сколь угодно близкой к плоскости, имеет всего две критические точки: максимум и минимум.

В действительности теорема о четырех вершинах, подобно теореме о четырех точках возврата каустик на поверхностях, относится к симплектической топологии. В частном случае римановой метрики справедливость теоремы о четырех вершинах восстанавливается, если определять вершины кривой следуюшим способом.

Выпустим из каждой точки нашей кривой геодезическую, перпендикулярную кривой и направленную во внутреннюю сторону. Точка пересечения такой нормальной геодезической с бесконечно-близкой нормальной геодезической называется сопряженной точкой (вдоль исходной нормали). Все эти сопряженные точки образуют каустику исходной кривой (огибающую семейства геодезических нормалей).

Теорема о четырех вершинах (в римановом варианте) утверждает, что каустика имеет не менее четырех точек возврата.

В случае обычной евклидовой плоскости геодезические нормали - это обычные нормали. Сопряженные точки - это центры кривизны кривой. Множество сопряженных точек (каустика) называется в этом случае фокальной кривой исходной кривой. Например, фокальная кривая эллипса - это астроида (рис. 2). Астроида имеет четыре точки возврата. Теорема о четырех вершинах утверждает, что это не случайно: фокальная кривая любой выпуклой кривой должна иметь не менее четырех точек возврата (по топологическим причинам, которые обсуждаются ниже и принадлежат симплектической топологии).

В случае евклидовой метрики точки возврата каустики случайно соответствуют экстремумам геодезической кривизны. В общем римановом случае "настоящие" вер- 


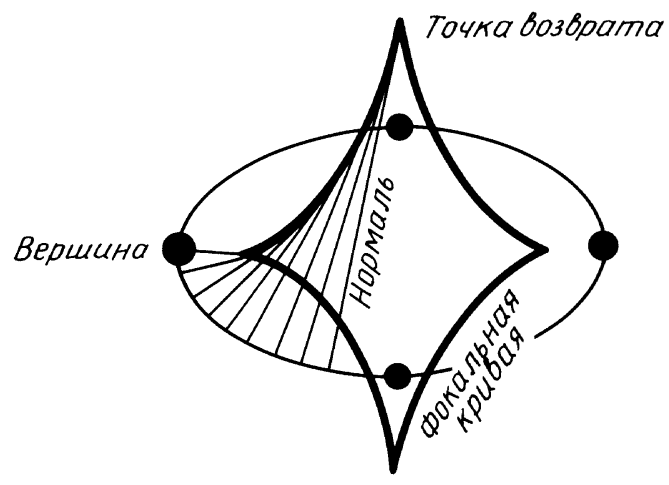

Рис. 2. Фокальная кривая эллипса

шины не определяются метрикой поверхности вблизи исходной кривой, а зависят еще от метрики вблизи ее нормали.

ПРИмеР 3. Ларморовские окружности. Рассмотрим движение заряженной частишы на евклидовой плоскости под действием перпендикулярного плоскости магнитного поля. Орбитой движения является ларморовская окружность кривизны $B / v$ (где $B$ - величина поля, $v$ - длина вектора скорости).

Рассмотрим семейство ларморовских окружностей частиц, выходящих из начальной точки по всем направлением с фиксированной энергией. Это - семейство окружностей фиксированного радиуса, проходящих через одну точку.

Начальная точка является (бесконечно-вырожденной) огибающей этого семейстBa.

Возмутим это семейство, заменив постоянную $B$ на близкую к ней функцию общего положения на плоскости (или заменив евклидову метрику плоскости близкой ей метрикой общего положения).

Бесконечно-вырожденная огибаюшая в исходной точке превратится после возмущения в замкнутую кривую (рис. 3).

ТЕОРемА. Оұибающая семейства возмущенных ларморовских орбит данной әнергии имеет не менее четырех точек возврата.

СлЕДСТВИЕ. Для любой метрики и для любого магнитного поля общего положения огибающая семейства малых ларморовских окружнностей (выходящих из одной точки с одинаковой и достаточно малой энергией), близкая к исходной точке, имеет не меньше четьрех точек возврата.

В частности, следствие применимо к семейству кривых большой постоянной геодезической кривизны римановой метрики обшего положения на поверхности.

Следствие выводится из теоремы при помощи изменения масштаба ( "разглядывания окрестности исходной точки в микроскоп"). Теорема непосредственно вытекает из того же факта симплектической топологии, что и теоремы о четырех точках возврата каустик предыдуших примеров. Симплектическая геометрия объединяет все эти факты (и факты нижеследующих примеров) в одну теорему, различными ипостасями которой являются теоремы всех наших примеров. 

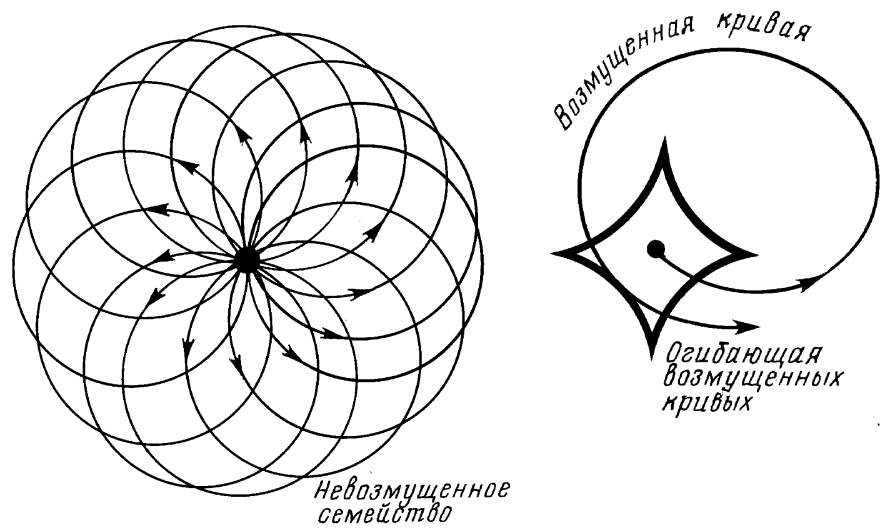

Рис. 3. Огибающая семейства ларморовских окружностей

ПримеР 4. Неголономные связи и каустики теории управления. Рассмотрим поле двумерных плоскостей общего положения в трехмерном пространстве. В окрестности точки обшего положения такое поле задается уравнением $d z=y d x$ (в подходяших локальных координатах $x, y, z)$. Такие поля называются также вполне неголономными связями, контактными структурами или распределениями Гейзенберга.

Согласно известной теореме Рашевского, из каждой точки обшего положения можно добраться до каждой близкой точки малым путем, двигаясь так, что скорость в каждый момент времени принадлежит плоскости поля.
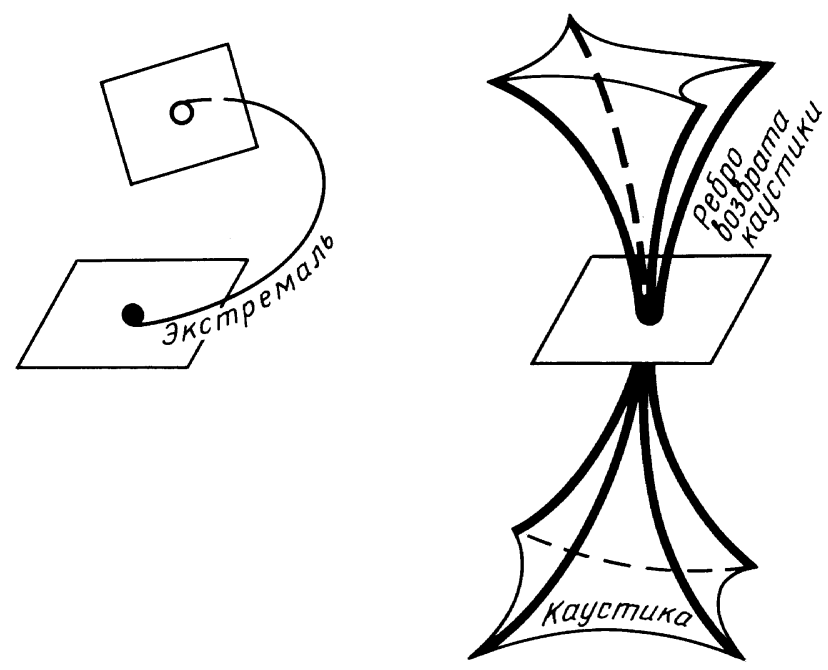

Рис. 4. Каустика управляемой системы

Зафиксируем в пространстве риманову метрику общего положения и постараемся двигаться так, чтобы длина пути (или действие) была минимальной. Соответствующие экстремали, выходящие из каждой точки общего положения, образуют трехпа- 
раметрическое семейство кривых. Огибающая этого семейства - поверхность, называемая каустикой исходной точки (рис. 4).

Теорема АГРАЧева [7]. Каустика точки общего положсения - поверхность с четырьмя ребрами возврата полукубического типа, касающимися друг друга в исходной точке (где все четыре ребра перпендикулярны плоскости допустимых направлений в смысле исследуемой римановой метрики).

Сечение каустики поверхностью, близкой к допустимой плоскости в исходной точке, имеет вид малой астроиды (кривой с четырьмя точками возврата полукубического типа). Линейный размер этой астроиды пропорционален квадрату расстояния от секушей поверхности до допустимой плоскости вдоль нормали в исходной точке. Точки каустики, близкие к исходной точке, достижимы за очень большое время, так как движение по соответствующей экстремали очень медленное.

Теорема Аграчева является обобщением теоремы о малой огибающей ларморовских окружностей, которая получается при специальном выборе римановой метрики (роль расстояния до допустимой плоскости играет в этом случае начальная энергия частиц).

ПримеР 5. Выворачивание волнового фронта. Рассмотрим волновой фронт на плоскости, распространяюшийся с единичной скоростью внутрь эллипса. Система мгновенных положений фронта - это система әквидистант исходного фронта. Она изображена на рис. 5.

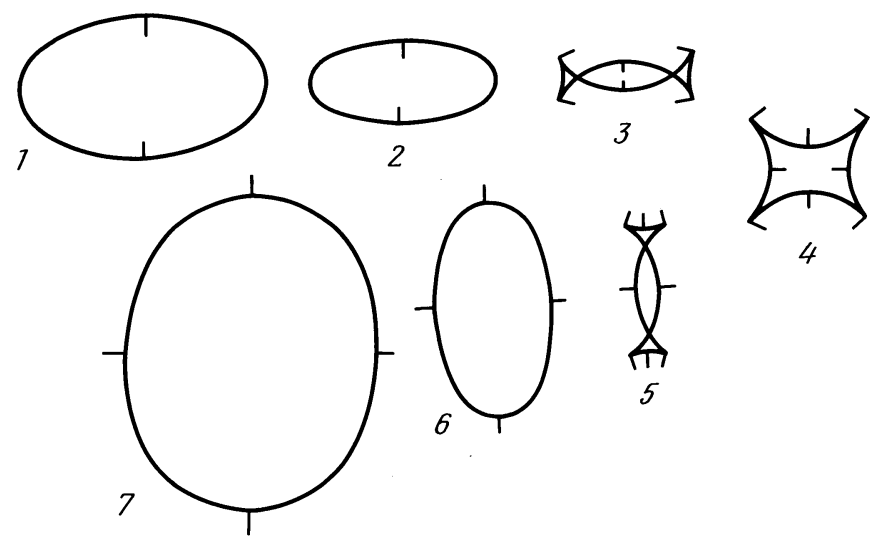

Рис. 5. Вьворачивание эллипса наизнанку

Эквидистанты, достаточно близкие к исходному фронту, гладкие. Затем на фронте возникают четыре точки возврата (полукубического типа). После нескольких перестроек фронт вновь становится гладким, но распространяется уже наружу: он вывернулся наизнанку.

Явление выворачивания можно заметить уже для фронта, первоначально имеюшего вид окружности. Но в этом случае переход от сжатия к расширению фронта происходит в момент полного коллапса фронта в точку. Коллапс фронта в точку - яв- 
ление бесконечно-вырожденное, происходящее из-за чрезвычайной симметричности задачи.

Для исходного фронта и метрики обшего положения выворачивание происходит не мгновенно, а проходя через несколько перестроек обшего положения, подобных изображенньм на рис. 5 .

ТЕОрема [8]. При выворачивании распространяющего волнового фронта общего положения, первоначально не слишком далекого от сжимающейся окружности на евклидовой плоскости, в некоторый промехуточный момент образуется волновой фронт с не менее чем четырьмя точками возврата.

ЗАмЕчАНИЕ. Из этого следует, в частности, что типичное выворачивание сопровождается не менее чем четырьмя перестройками рождения или уничтожения пары точек возврата фронта (на рис. 5 их ровно четыре: рождение двух малых треугольников на этапе 2-3 и затем смерть двух других на этапе 5-6).

Траектории точек возврата перестраивающейся системы фронтов образуют каустику семейства. Например, каустика системы эквидистант кривой на евклидовой плоскости - это в точности фокальная кривая исходного фронта. Рождение и смерть точек возврата фронтов соответствуют точкам возврата каустики. Предыдущее замечание означает, что каустика системы выворачивающихся фронтов общего положения (достаточно близкой к стандартному выворачиванию окружности) имеет не менее четырех точек возврата.

Теорема о вьворачивании является в этом смысле обобшением теоремы о четырех вершинах.

ЗАмечАниЕ. Вопрос о том, насколько сильно выворачивание может отличаться от стандартного, чтобы утверждение о четырех точках возврата промежуточного фронта оставалось верным, представляется очень интересным. На рис. 6 изображено выворачивание в однопараметрическом семействе общего положения фронтов на плоскости, для которого все фронты имеют не более двух точек возврата. Число рождений и уничтожений пар точек возврата в процессе выворачивания (число точек возврата каустики) в этом примере равно 2 (рождение на этапе $1-2$, смерть на этапе 6-7).

Явление этого рода невозможно в системе эквидистант. Действительно, между этапами 3 и 4 должна произойти перестройка мгновенного самокасания фронта, причем направления коориентирующих нормалей касающихся ветвей совпадают. Такое же самокасание должно произойти между этапами 4 и 5.

ОПРЕДЕЛЕНИЕ. Касание с совпадением направления коориентирующих нормалей назьвается опасным.

ПРЕДЛОЖЕНИЕ. В системе эквидистант гладкой кривой (или, более общим образом, в системе первоначально гладких волновых фронтов, распространяющихся в соответствии с уравнением Гамильтона-Якоби) опасное самокасание невозможно.

ДокАЗАТЕльство. В соответствии с принципом Гюйгенса элементы распространяющегося фронта эволюционируют автономно (независимо друг от друга), подчиняясь гамильтоновой динамической системе (каноническим обыкновенным дифференциальным уравнениям Гамильтона). По теореме единственности опасное самокасание 

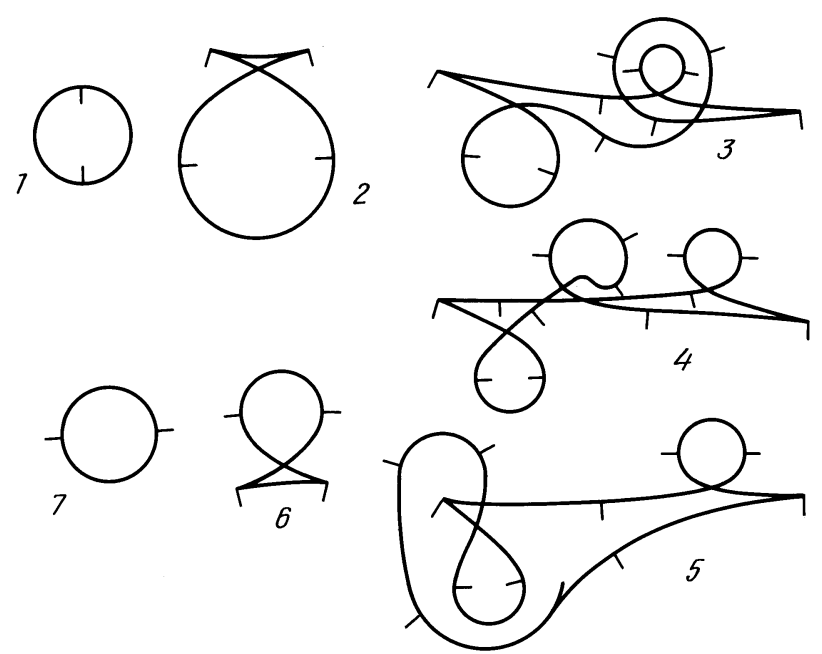

Рис. 6. Выворачивание фронта с не более чем двумя точками возврата

не может возникнуть в процессе эволюции первоначально гладкого фронта: если оно присутствует в какой-либо момент, то оно должно было бы присутствовать уже в начальньй момент.

ГИПотеЗА 1. В однопараметрическом общего положения семействе волновых фронтов на плоскости без опасных самокасаний, реализующем выворачивание гладкого замкнутого фронта, присутствует фронт с не менее чем четьрьмя точками возврата.

Эта гипотеза близка к следуюшему утверждению.

ГИПоТЕЗА 2. Всякое однопараметрическое семейство общего положсения плоских замкнутых кривых с двумя точками возврата, соединяющее кривые 2 и 6 (рис. 6), содержит фронт с опасным самокасанием.

Попытки доказательства этой гипотезы привели к построению теории инвариантов плоских кривых и волновых фронтов (см. [8]-[23]). Эта теория тесно связана с теорией инвариантов Васильева узлов [24], с квантовой теорией поля, интегральной геометрией, статистической физикой и алгебраической геометрией. Однако гипотезы 1 и 2 остаются недоказанньми и неопровергнутыми.

Инфинитезимальным вариантом всех предыдущих и многих последующих топологических теорем является описываемый ниже факт теории рядов Фурье и его обобщения.

\section{§3. Теорема Гурвица-Келлога-Табачникова типа Штурма}

Разложим $2 \pi$-периодическую функцию в ряд Фурье

$$
f(x)=\sum_{n \geqslant N} a_{n} \cos n x+b_{n} \sin n x .
$$


Tеорема (см. [25], [26], [6]). Число нулей функции на окружности не меньие числа нулей первой гармоники, входящей в ее ряд Фурве с ненулевым коэффициентом:

$$
\#(x \bmod 2 \pi: f(x)=0) \geqslant 2 N .
$$

При $N=1$ условие $n \geqslant N$ означает, что функция $f$ в среднем равна нулю, т.е. является производной некоторой функции по окружности: $f=d g / d x$.

Утверждение теоремы в этом случае преврашается в неравенство Морса для функций на окружности: число критических точек функции $g$ на окружности не меньше 2 .

При $N=2$ теорема утверждает, что функиия, ортогональная функииям $1, \sin x$ $u \cos x$, на окружности имеет не менее четырех нулей (другая формулировка: функиия, ортогональная синусу и косинусу, имеет на окружности не менее четырех критических точек).

Вычисления по теории возмушений показьвают, что все топологические теоремы примеров 1-5 вьше (как и следуюших ниже примеров $\S \S 3$ и 4) сводятся именно к этому частному случаю $N=2$ сформулированной вьше теоремы для "бесконечномалых возмущений" стандартных интегрируемых примеров “коллапса" (для геодезических на маловозмушенной стандартной сфере в примере 1, для плоских кривых, близких к евклидовой окружности, в примере 2 , для малых возмушений стандартных ларморовских окружностей в примере 3 и т. д.).

Имеется много различных доказательств теоремы (допускаюших также различные обобщения). Одно из самых коротких (и наименее понятных) доказательств комплексное. Для простоты я приведу его для случая, когда $f$ - тригонометрический многочлен:

$$
f(x)=\operatorname{Re} F(z), \quad F(z)=\sum_{n \geqslant N} A_{n} z^{n}, \quad z=e^{i x} .
$$

Индекс функции $F$ на единичной окружности $|z|=1$ равен

$$
(\Delta \arg F(z)) /(2 \pi)=\text { (число нулей } F \text { в круге }|z| \leqslant 1) \geqslant N \text {. }
$$

Следовательно, число нулей вешественной части $F$ на окружности не меньше $2 N$ (рис. 7), что и требовалось доказать.

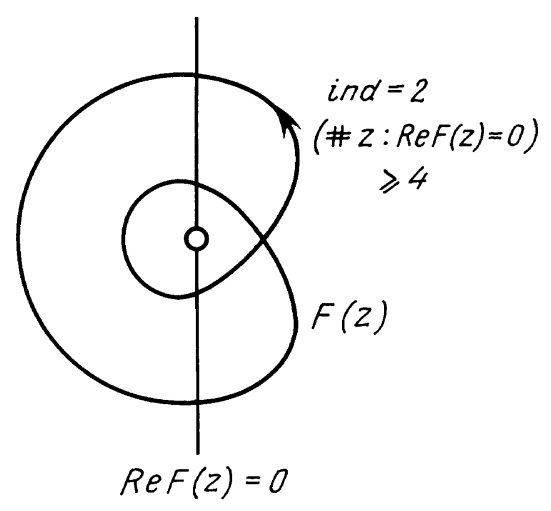

Рис. 7. Четыре нуля вешественной части кривой индекса два 
Другое доказательство доставляется уравнением теплопроводности. Примем $f$ за начальное условие и будем следить за временной эволюцией решения. Высшие гармоники затухнут быстрее чем первая присутствующая в ряду Фурье с ненулевым коэффициентом. Поэтому, начиная с некоторого момента времени, число нулей решения будет в точности равно $2 N$.

Между тем число нулей в процессе эволюции не увеличивалось (как это легко вывести из принципа максимума или его несложного обобщения). Следовательно, исходное число нулей не меньше $2 N$.

Еше одно доказательство (проходяшее для любой чебьшевской системы функций вместо тригонометрической системы) имеется в [27].

В простейшем (но как раз нужном нам сейчас) случае $N=2$ оно сводится к следуюшему. Предположим, что функция $f$, ортогональная единице, синусу и косинусу, имеет меньше четырех нулей. Тогда она меняет знак на окружности не более двух раз. Поэтому существует линейная комбинация $l=a \cos x+b \sin x+c$, меняющая знак в тех же точках, что и $f$. Произведение $f l$ не меняет знака. Это противоречит ортогональности $l$ и $f$. Поэтому $f$ имеет не меньше четырех перемен знака.

ПримеР 1. Теорема о теннисном мяче. Рассмотрим на сфере несамопересекающуюся замкнутую кривую, т.е. гладкое вложение $S^{1} \rightarrow S^{2}$.

ОПРЕДЕЛЕНИЕ. Вложение назьвается точным, если его образ делит сферу на две части равной площади (рис. 8).

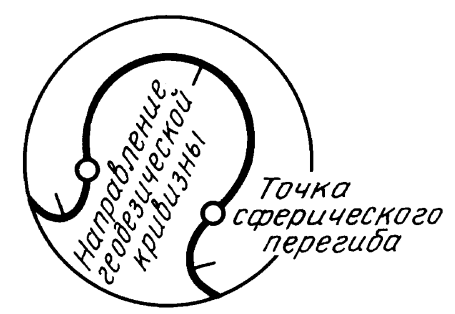

Рис. 8. Теорема о теннисном мяче

ТЕОрема [8]. Точно вложенная в сферу кривая имеет не менее четырех точек сферического перегиба (әде кривизна кривой обращается в нуль).

Примером точно вложенной кривой является экватор. Все его точки являются точками сферического перегиба. Рассмотрим малое возмущение экватора. Оно, естественно, определяется вешественной функцией на экваторе (расстоянием $f$ до возмушенной кривой по нормали).

Точки сферического перегиба определяются (в первом приближении) из условия $f^{\prime \prime}+f=0$. Условие точности означает, что среднее значение $f$ на окружности равно нулю. Поэтому сушествование не менее четырех точек сферического перегиба для бесконечно-малых возмущений экватора вытекает из теоремы Гурвища.

Теорема о теннисном мяче утверждает, что результат остается верньм и для конечных возмушений, даже очень больших: достаточно, чтобы точная кривая не самопересекалась. Возмущенная кривая не обязательно трансверсальна меридианам. 
Соответствующая ей функция на экваторе, вообще говоря, многозначна. Таким образом, теорему о теннисном мяче моэсно рассматривать как обобщение теоремь Гурвица на случай многозначных функций.

ЗАмЕчАниЕ. Иммерсированные (т.е. самопересекающиеся) точные кривые на сфере могут иметь всего лиш две точки сферического перегиба.

Точность иммерсированной в сферу площади $4 \pi$ окружности определяется так: интеграл формы площади по 2-цепи, ограниченной кривой, должен быть равен $2 \pi$ $(\bmod 4 \pi)$.

Пример точной иммерсии с всего двумя точками сферического перегиба (из [28]) изображен на рис. 9 (где $A+C=B$ ).

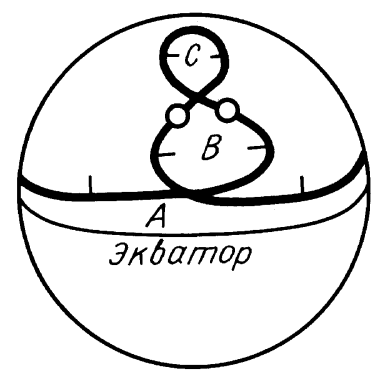

Рис. 9. Точная иммерсия с двумя точками сферического перегиба

ОПРЕДЕЛЕНИЕ. Псевдофункиией называется точная иммерсия окружности в сферу, которую можно соединить с вложением экватора регулярной гомотопией в классе точных иммерсий, для которых никакая из входящих в гомотопию кривых не имеет замкнутой петли (отличной от всей кривой), ограничивающей кратную $2 \pi$ площадь.

ЗАмЕчАниЕ. Отсутствие таких петель означает, что соответствующая лежандрова кривая в $S^{3}$ является лежандровым узлом (не самопересекается), т.е. что лежандров тип узла, соответствующего продеформированной лагранжевой точной кривой в $S^{2}$ тривиален (такой же, как тип лежандрова узла, соответствующего экватору $S^{2}$ ). Подробности см. в [12].

ГИПоТЕЗА. Псевдофункиия имеет не менее четырех точек сферического перегиба.

ПримеР 2. Теорема о четырех положениях равновесия. Рассмотрим однородную пластину, ограниченную плоской вьпуклой кривой. Следующий результат является классическим.

ТЕОРема. Вертикально поставленная на горизонтальный стол пластина имеет не менее четырех полохсений равновесия.

Эквивалентная формулировка:

ТЕОРЕМА. Из чентра масс области, ограниченной выпуклой кривой на плоскости, мохнно опустить на кривую не менее четырех перпендикуляров. 
ДокАЗАТЕльство. Обозначим через $x=r \cos \varphi, y=r \sin \varphi$ декартовы и полярные координаты на плоскости с началом в центре масс. Пусть $r=R(\varphi)$ - уравнение кривой в полярных координатах. Условие, что начало координат - центр масс, означает равенство нулю интегралов момента

$$
I_{x}=\iint x d x d y, \quad I_{y}=\iint y d x d y
$$

Вычисляя эти интегралы в полярных координатах, мы находим

$$
I_{x}=\iint r \cos \varphi r d r d \varphi=\int \frac{R^{3}}{3} \cos \varphi d \varphi, \quad I_{y}=\int \frac{R^{3}}{3} \sin \varphi d \varphi
$$

Так как эти интегралы равны нулю, функция $R^{3}$ ортогональна синусу и косинусу. По теореме Гурвица она имеет не менее четырех критических точек.

Значит, и функция $R=R(\varphi)$ имеет на окружности не менее четырех критических точек, что и требовалось.

ЗАмечАниЕ. Доказательство доставляет также многочисленњые обобщения теоремы. Например, вместо выпуклости достаточна звездность области. Однородную плотность можно заменить (положительной) функцией от $r$, и т. д.

ПримеР 3. Теорема о четырех точках уплощения. Рассмотрим выпуклую кривую положительной кривизны на плоскости, лежащей в трехмерном пространстве. Возмутим эту кривую, так чтобы получилась пространственная кривая. Возмущение предполагается здесь достаточно мальм вместе с производными ниже третьего порядка.

ТЕОРемА. Кручение возмущенной кривой обращается в нуль не менее, чем в четырех точках.

ОПРЕДЕЛЕниЕ. Точки, где кручение обращается в нуль, называются точками уплощения.

Следуюшее утверждение эквивалентно сформулированному:

ТЕОРема. Предположим, что проекиия пространственной кривой на плоскость - вложенная выпуклая замкнутая кривая положстельной кривизны. Тогда исходная кривая имеет не менее четырех точек уплощения.

ДокАЗАТЕЛЬСТво. Точки уплощения переходят при проективных преобразованиях в точки уплощения, так как это точки необычно тесного касания кривой с соприкасающейся плоскостью.

Сжатие вдоль направления проектирования делает исходную пространственную кривую сколь угодно близкой к ее плоской проекции. Поэтому вторая теорема следует из первой.

Обратно, проекция слабо возмушенной выпуклой кривой положительной кривизны на исходную плоскость - выпуклая кривая положительной кривизны. Поэтому первая теорема следует из второй. 
ЗАмЕчАниЕ. Теорема о четырех вершинах плоской кривой следует из любой из сформулированных теорем о пространственных кривых.

Действительно, стереографиическая проекция плоскости на сферу достаточно большого радиуса переводит исходную вьпуклую кривую на плоскости в сколь угодно близкую к ней пространственную кривую. Точки уплощения полученной пространственной кривой являются образами вершин исходной плоской кривой (так как прообразы плоских сечений сферы - окружности на исходной плоскости). Полученная пространственная кривая имеет по меньшей мере четыре точки уплошения по сформулированной теореме. Значит, исходная плоская кривая имеет не менее четырех вершин. Обобшение классической теоремы о четырех точках уплошения на кривые в многомерных пространствах обсуждается в § 9 ниже.

ПримеР 4. Теорема Жиса о нулях шварцевой производной. Проективная прямая $\mathbb{R P}^{1}$ диффеоморфна окружности $S^{1}$.

Рассмотрим гладкое отображение окружности на окружность как отображение проективной прямой на проективную прямую.

ОПРЕДЕЛЕНИЕ. Точка называется проективно стационарной для отображения проективной прямой на проективную прямую, если шварцева производная отображения в этой точке равна нулю.

Иными словами, проективно стационарная точка отображения - это точка, в которой оно необычно хорошо аппроксимируется проективным отображением.

Проективные отображения прямой на прямую (дробно-линейные функции) образуют трехмерное многообразие. В каждой точке прямой-прообраза можно подобрать проективное отображение так, чтобы совпали с заданными значения функции и первых двух производных. Проективно стационарные точки - это те, в которых совпадают еше и третьи производные исходного отображения и его проективной (т.е. дробно-линейной) аппроксимации.

ЗАмечАниЕ. Обычные стационарные (критические) точки функции - это точки, в которых приближение подходящей постоянной функцией правильно описывает не только функцию, но и ее первую производную.

ТЕоРема ЖИСА. Диффеоморфизм проективной прямой на проективную прямую имеет не менее четырех точек проективной стационарности.

ЗАмечАниЕ 1. "Инфинитезимальное" вычисление для малых возмущений тождественного (а значит, и любого проективного) отображения приводит после небольших вычислений к случаю $N=2$ теоремы Гурвица.

Поэтому теорему Жиса можно рассматривать как некоторое обобщение случая $N=2$ теоремы Гурвица.

ЗАмечание 2. В. Овсиенко и С. Табачников [29] вывели теорему Жиса из теоремы о теннисном мяче.

ЗАмечаниЕ 3. Как мне указал М.Э. Казарян, теорема Жиса допускает следующую переформулировку (с которой Жис, кажется, и начинал): 
ТЕОРемА. Замкнутая кривая на поверхности однополостного гиперболоида, пересекающая под ненулевыми углами по одному разу все его прямолинейные образующие, имеет в трехмерном проективном пространстве не менее четырех точек уплощения.

ДОКАЗАТЕЛЬСТВО ЭКВИВАЛЕНТНОСТИ. Однополостньй гиперболоид можно рассматривать как $\mathbb{R} \mathrm{P}^{1} \times \mathbb{R} \mathrm{P}^{1}$, так как каждые две образующие разных семейств пересекаются в одной точке. График диффеоморфизма $\mathbb{R} \mathrm{P}^{1} \rightarrow \mathbb{R P}^{1}$, рассматриваемьй как кривая на поверхности гиперболоида - то же, что замкнутая кривая, трансверсально пересекаюшая по одному разу все прямолинейные образуюшие обоих семейств.

Плоские неособые сечения гиперболоида (не касающимися гиперболоида плоскостями) - это в точности графики проективных отображений. Точки уплощения графика - это в точности точки, где диффеоморфизм необычно тесно аппроксимируется подходящими проективными отображениями.

Число проективно-стационарных точек диффеоморфизма равно поэтому числу точек уплошения его графика.

\section{§4. Тригонометрические аппроксимации}

Здесь мы рассмотрим еще одно приложение теоремы Гурвица типа Штурма в теории тригонометрических многочленов (или, общее, чебьшевских систем).

Критическая точка функции - это точка, в окрестности которой подходящая постоянная дает аппроксимацию степени 2 или вьше (так что погрешность аппроксимации менњше $C t^{2}$, где $t$ - расстояние до рассматриваемой точки, а $C$-постоянная). Аналогично определяется аппроксимация степени $n$ функции $f$ функцией $p$ в точке $x$ :

$$
|f(y)-p(y)| \leqslant C t^{n}, \quad t=|y-x| .
$$

Tеорема 1 [30]. Гладкая функиия на окружности $\{x \bmod 2 \pi\}$ допускает anпроксимацию порядка $2 n+2$ тригонометрическими многочленами степени не выше $n$ не менее, чем в $2 n+2$ точках.

ПримеР. При $n=0$ это - неравенство Морса: гладкая функция на окружности имеет не менее двух критических точек.

Нижеследуюшая теорема 2 показывает, что наша теорема 1 является обобшением этого неравенства Морса на старшие производные. Пространство тригонометрических многочленов степени не выше $n$ имеет размерность $2 n+1$. Поэтому аппроксимация тригонометрическим многочленом степени не выше $n$ порядка $2 n+1$ возможна в любой точке $x$.

Точки, в которых возможна аппроксимация тригонометрическими многочленами степени не вьше $n$ более высокой точности, назьваются $n$-экстатическими точками данной функции. Наша теорема утверждает, например, что число 1-экстатических точек функции на окружности не меньше четырех.

Эта четверка - та же, что и во всех предыдущих примерах. Чтобы в этом убедиться, рассмотрим следующий алгоритм нахождения $n$-экстатических точек функции $f$. 
ТЕОРема 2. Точка $x$ является $n$-әкстатической для $2 \pi$-периодической функичи $f$, если и только если в этой точке обращается в нуль функиия Lf, где L дифференииальный оператор

$$
L=\partial\left(\partial^{2}+1\right)\left(\partial^{2}+4\right) \ldots\left(\partial^{2}+n^{2}\right) .
$$

ДОКАЗАТЕЛЬСТВо ТЕОРЕМЫ 2 (ср. [30] и [27]). ПредПоложим, что $f-p$ (где $p$ - тригонометрический многочлен степени не вьше $n$ ) имеет в точке $x$ нуль порядка вьше $2 n+1$. Тогда $L(f-p)=0$ в точке $x$ (поскольку порядок $L$ равен $2 n+1)$. Но $L p \equiv 0$, так как $\partial^{2}+k^{2}$ уничтожает гармоники $\sin (k y)$ и $\cos (k y)$. Значит, $(L f)(x)=0$.

Обратно, пусть $(L f)(x)=0$, а $p$ - тригонометрический многочлен степени не выше $n$, аппроксимируюший $f$ в точке $x$ с порядком аппроксимашии $2 n+1$. Тогда производные $f-p$ до порядка $2 n$ включительно в точке $x$ совпадают. Убедимся, что производные порядка $2 n+1$ в точке $x$ тогда тоже совпадают.

Мы имеем $L=\partial^{2 n+1}+L^{\prime}$, где $L^{\prime}$ - оператор меньшего, чем $2 n+1$, порядка. Теперь мы получаем

$$
L(f-p)(x)=\partial^{2 n+1}(f-p)(x)+L^{\prime}(f-p)(x) .
$$

Левая часть равна нулю по предположению $(L f)(x)=0$, так как $L p \equiv 0$. Второе слагаемое справа равно нулю, так как производные менњшего, чем $2 n+1$, порядка функций $f$ и $p$ в точке $x$ одинаковы. Значит, производные порядка $2 n+1$ функций $f$ и $p$ в точке $x$ совпадают, так что эта точка $-n$-экстатическая. Теорема 2 доказана.

ДокАЗАТЕЛЬСТво ТЕОРЕмы 1 . Функция $L f$ ортогональна всем гармоникам

$$
1, \cos x, \sin x, \ldots, \cos (n x), \sin (n x) .
$$

По тереме Гурвица она имеет не менее $2 n+2$ нулей на окружности. Эти нули являются $n$-экстатическими точками для $f$ по уже доказанной теореме 2 . Теорема 1 доказана.

Мы видим, что $n$-экстатические точки являются обобщениями критических, только оператор первого порядка $\partial$, входящий в определение критической точки, заменяется оператором $L$ порядка $2 n+1$.

ЗАмЕчАниЕ. Теоремы 1 и 2 допускают много обобщений.

ОПРЕДЕЛЕНИЕ. Система функций $u_{1}, \ldots, u_{N}$ назьвается чебъиевской, если число нулей любой их нетривиальной линейной комбинации (учитывая кратности) меньше чем $N$.

Тригонометрическую систему функций в теоремах 1 и 2 можно заменить любой другой чебышевской системой функций на окружности, $u_{1}, \ldots, u_{2 n+1}$. Пусть $L-$ дифференпиальный оператор порядка $2 n+1$, имеюший эти функции фундаментальной системой решений. Тогда $n$-экстатические точки $x$ функции $f$ (где она необычно хорошо аппроксимируется линейными комбинациями функций $u_{i}$ ) определяются из уравнения $\left(L^{*} f\right)(x)=0$, где $L^{*}-$ сопряженный с $L$ (по Лагранжу) оператор.

Оператор $L^{*}$, подобно $L$, имеет чебьшевскую фундаментальную систему решений. Функция $L^{*} f$ имеет не менее $2 n+2$ нулей по обобщенной теореме Гурвица (Келлога, Табачникова). 
Аналогичные результаты имеют место и для чебышевских систем из четного числа функций (точнее, для сечений расслоения Мёбиуса - единственного нетривиального одномерного вещественного расслоения над окружностью). Такие мёбиусовские функции можно рассматривать как антипериодические функции на двулистно накрываюшей окружности, $f(x+\pi) \equiv-f(x)$ (причем под числом нулей можно понимать число нулей на интервале $[0, \pi))$.

Подробности см. в [27], [30]. Отсутствие теорем 1 и 2 в литературе кажется мне удивительным.

\section{§5. Лагранжевы пересечения в симплектической топологии}

Чтобы объяснить топологический смысл описанных вьше обобщений неравенств Морса для высших производных, начнем с обобщения обычных неравенств Морса на случай многозначных ${ }^{1}$ функций - с теории лагранжевых пересечений - в простейшем случае функций на окружности.

Рассмотрим фазовоепространство, конфигурационным многообразием которого является окружность $S^{1}=\{q \bmod 2 \pi\}$. Это фазовое пространство $T^{*} S^{1}$ (пространство кокасательного расслоения окружности) представляет собой цилиндр $S^{1} \times \mathbb{R}=$ $\{q \bmod 2 \pi, p \in \mathbb{R}\}$, снабженньй формой действия $\alpha=p d q$.

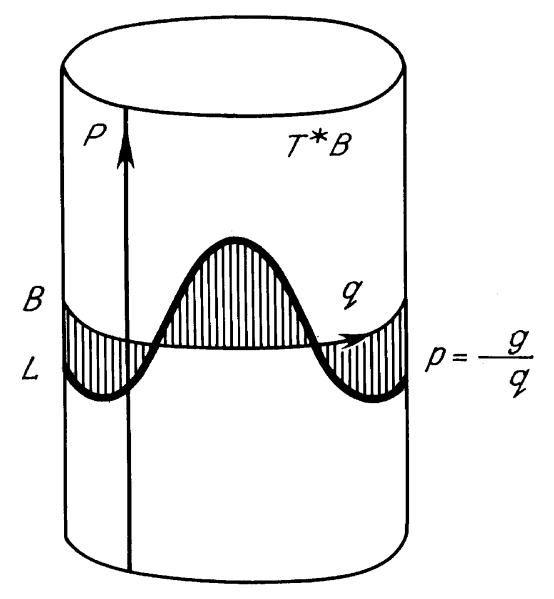

Рис. 10. Точное лагранжево сечение

Функции $g$ на окружности сопоставляется кривая на цилиндре (рис. 10)

$$
p=f(q), \quad f(q)=\frac{\partial g}{\partial q}
$$

Ориентированная площадь между этой кривой и экватором $p=0$ равна нулю.

\footnotetext{
${ }^{1}$ Имеются в виду не замкнутые 1-формы, а настоящие многозначные функции с ветвлением, вроде $\sqrt{x}$.
} 
ОПРЕДЕЛЕНИЕ. Подмногообразие средней размерности $n$ в пространстве кокасательного расслоения $T^{*} B^{n}$ конфигурационного $n$-мерного многообразия, заданное функцией $f: B^{n} \rightarrow \mathbb{R}$ по формуле (1), называется точным лагранжсевым сечением расслоения.

С физической точки зрения точное лагранжево многообразие можно представить себе как потенциальное поле скоростей на $B$ (если отождествить кокасательные векторы $p$ с касательными $\dot{q}$, например, при помощи какой-либо римановой метрики на $B$ ).

2-фороа $\omega=d \alpha\left(=\sum d p_{i} \wedge d q_{i}\right)$ фазового пространства тождественно равна нулю на лагранжевом сечении (1), так как на нем $\alpha=d f$.

ОПРЕДЕЛЕниЕ. Многообразие $M^{2 n}$, снабженное замкнутой невырожденной в каждой точке дифференциальной 2-формой $\omega$, называется симплектическим многообразием. Диффеоморфизм одного симплектического многообразия на другое назьвается симплектоморфизмом, если он переводит одну из симплектических форм в другую.

ПРИМеР. Рассмотрим сферу $x^{2}+y^{2}+z^{2}=1$, вписанную в цилиндр $x^{2}+y^{2}=1$ в трехмерном евклидовом пространстве.

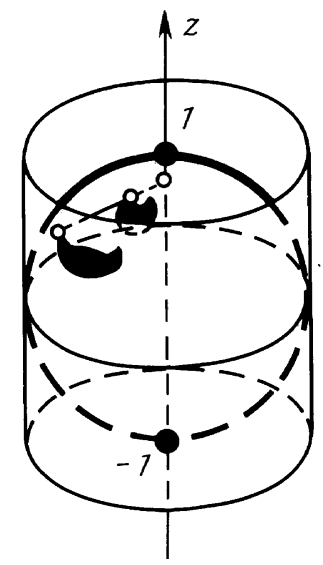

Рис. 11. Симплектоморфизм Архимеда

ЛЕмма АрхимедА. Отображсене сферы без полюсов на иилиндрическую область $|z|<1$, заданное горизонтальнымм лучами (рис. 11), выходящими из точек вертикальной оси z, является симплектоморфизмом.

ОПРЕДЕЛЕНИЕ. Лагранжевым подмногообразием симплектического многообразия $\left(M^{2 n}, \omega\right)$ называется подмногообразие средней размерности $n$, ограничение на которое симплектической 2-формы $\omega$ обрашается тождественно в нуль.

ПримЕР. Всякая кривая на поверхности цилиндра $T^{*} S^{1}$ лагранжева.

Лагранжево подмногообразие $L^{n}$ фазового пространства $\left(T^{*} B^{n}, \omega=d \alpha\right)$ назьвается точным, если ограничение формы действия на него является полным дифференциалом некоторой функции, $\left.\alpha\right|_{L^{n}}=d S, S: L^{n} \rightarrow \mathbb{R}$. 
ПримеР. Вложенная в поверхность цилиндра $S^{1} \times \mathbb{R}$ замкнутая кривая точна, если и только если (ориентированная) плошадь между этой кривой и экватором $p=0$ равна нулю: $\oint p d q=0$.

Точное лагранжево подмногообразие пространства кокасательного расслоения $T^{*} B^{n}$, получаюшееся из нулевого сечения $(p=0)$ регулярной гомотопией ${ }^{2}$ в классе точных лагранжевых подмногообразий, может рассматриваться как график производной многозначной функиии на $B^{n}$.

Физически лагранжево многообразие-это многопотоковое потенциальное течение. В таком течении через одну и ту же точку конфигурационного многообразия $B$ могут одновременно проходить по разным направлениям несколько потенциальных потоков. Эта ситуация реализуется в пылевидных средах или в звездных потоках и находит применение в астрофизике и в космологии.

Неравенство Морса для многозначных функций на окружности, задающихся точными лагранжевыми кривыми на поверхности цилиндра, - это следующий (очевидный) факт.

ТЕОРемА. Точная вложенная в поверхность иилиндра замкнутая кривая пересекает экватор (нулевые сечения $p=0$ ) не менее, чем в двух точках.

ДокАЗАТЕльство. В противном случае кривая была бы расположена в верхнем (либо в нижнем) полуцилиндре и интеграл от $p d q$ вдоль нее был бы положителен (либо отрицателен).

В 1965 году я предположил, что аналогичное "неравенство Морса" справедливо и для многозначных функций на любом многообразии (см. [28], [31]).

ГИПОТЕЗА О ЛАГРАНЖЕВЫХ ПЕРЕСЕЧЕНИЯХ. Точное лагранжсево подмногообразие $L^{n}$ фазового пространства $T^{*} B^{n}$, получающееся из нулевого сечения при помощи регулярной гомотопии в классе точных лагранжевых многообразий, пересекает нулевое сечение $B^{n}$ в не меньшем числе точек, чем минимальное число критических точек гладкой функиии на конфигурационном многообразии $B^{n}$.

В частности, число точек пересечения $L^{n} \cap B^{n}$ для $L^{n}$ общего положения не меньше, чем сумма чисел Бетти многообразия $B^{n}$.

Эта гипотеза (а также аналогичные гипотезы из [28] и [31] для чисел неподвижных точек симплектоморфизмов, обобщаюшие "последнюю геометрическую теорему” Пуанкаре об отображениях кольца в себя, сохраняющих площади) была доказана для большинства встречающихся на практике многообразий: торов, произведений сфер и т. д.

Начиная с пионерской работы Конли и Цендера 1983 г. [32], этому вопросу посвящен цельй ряд статей Шаперона, Лауденбаха, Сикорава, Чеканова, Громова, Флоера, Хофера, Саламона, Гивенталя, Оно и др., [33]-[41].

Быть может, наиболее известным следствием этой деятельности является теория "гомологий Флоера" трехмерных гомологических сфер (эйлерова характеристика этих гомологий - инвариант Кассона, см. [42], [43]).

\footnotetext{
${ }^{2}$ Регулярная гомотопия гладких отображений (вложений, иммерсий, т.е. вложений с самопересечениями) - это путь в соответствующем пространстве гладких отображений, соединяющий одно отображение с другим.
} 
Хотя первоначальная гипотеза в полном объеме и остается, насколько мне известно, недоказанной и неопровергнутой и сегодня, следствие, относящееся к числам Бетти, доказано (см. [33], [34]):

ТЕОРЕМА. Число точек пересечения точного лагранжсева подмногообразия общего положсения с нулевым сечением кокасательного расслоения компактного многообразия остается ограниченным снизу суммой чисел Бетти многообразия при деформации нулевого сечения, оставляющей его точным лагранжсевым подмногообразием фазового пространства.

Эта теорема превращается в обычное неравенство Морса в случае мальх (с производными) деформаций нулевого сечения, так как тогда продеформированное лагранжево многообразие остается точным лагранжевым сечением. Его точки пересечения с нулевьм сечением - это критические точки соответствующей функции-потенциала, заданной на нулевом сечении.

Если же деформация не является малой, то продеформированное многообразие может и не быть сечением. В этом случае потенциал $S=\int p d q$ оказывается однозначной функцией не на нулевом сечении, а лишш на продеформированном многообразии $L$. На нулевом же сечении его можно рассматривать лишш как многозначную функцию $p(q)$.

Точки пересечения продеформированного многообразия с нулевым сечением $p=0$ остаются критическими точками потенциальной функции $S$ на $L$. Но у этой функции на $L$ появляются также лишние критические точки (где $\left.p d q\right|_{L}=0$, хотя $p$ не 0 ). В этом случае обычное неравенство Морса для функций на $L$ оценивает снизу лишш общее число интересующих нас критических точек (где $p=0$ ) и лишних критических точек. Сформулированная вьше (и весьма нетривиальная) теорема утверждает, что неравенство Морса вьполняется уже для одних лишш точек пересечения с нулевьм сечением, не учитывая лишние критические точки.

Идея доказательства состоит в том, чтобы перестроить потенциальную функцию так, чтобы нужные нам критические точки остались, а лишние исчезли. Это достигается своеобразной “стабилизацией” - переходом к функциям на расслоенном над $L$ многообразием бо́льшей размерности (к так назьваемым “производящим семействам").

Сформулированные в предыдуших параграф̆ах гипотезы находятся в таком же отношении к теореме Гурвица типа Штурма, в каком сформулированная вьше теорема о лагранжевых пересечениях находится к теории Морса. До тех пор, пока возмушенные объекты (метрики на сфере в задаче Якоби примера $1 \S 2$, пространственные кривые в теории уплощений примера $3 \S 3$ и т. д.) остаются достаточно близкими к невозмущенным, оценки снизу для чисел точек возврата, точек уплощения и т. д. можно получить из теоремы Гурвища (прямо доставляющей эти оценки для бесконечно-малых возмущений).

В случае бо́льших возмущений оценку числа особенностей снизу иногда удается доказать (например, для теоремы о теннисном мяче), но в большинстве случаев она остается гипотетической (например, в случае псевдофункций того же примера $1 \S 3$ ). 


\section{§6. Лежандровы зацепления в контактной топологии}

Пример рис. 9 показьвает, что для справедливости оценки числа особенностей снизу необходимы некоторые ограничения на деформации. На характер этих ограничений указывает теория точек пересечения точных лагранжевых иммерсий ( самопересекающихся подмногообразий), в которой этот пример впервые и возник в 1965 г. [28].

При деформации точной лагранжевой кривой на поверхности цилиндра могут возникать самопересечения. Условие точности $(\oint p d q \equiv 0)$ имеет смысл и для иммерсированных подмногообразий (интегрирование ведется по любой замкнутой кривой на гладком отображаемом многообразии, а не на образе иммерсии - например, в случае иммерсированной лагранжевой кривой условие состоит в том, что интеграл вдоль всей кривой равен нулю).

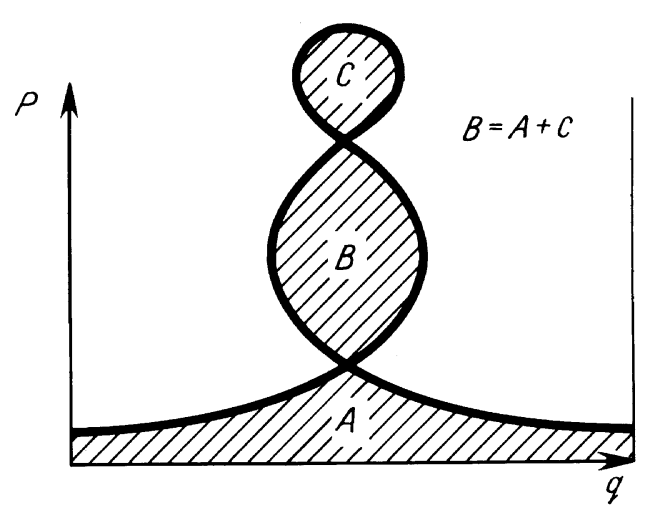

Рис. 12. Точная лагранжева иммерсия, не пересекающая нулевое сечение

На рис. 12 условие точности состоит в равенстве $B=A+C$ для (положительных) плошадей. Ясно, что иммерсированная точная лагранжева кривая в этом примере вовсе не пересекает нулевое сечение (хотя и получается из него регулярной гомотопией в классе точных лагранжевых иммерсий окружности в цилиндр).

Ю. В. Чеканов заметил, что подобный пример становится невозможным, если потребовать, чтобы в процессе деформации не менялся лежандров узел, соответствуюший точному лагранжеву подмногообразию [35], [44]. Чтобы сформулировать соответствуюшее обобщение теории Морса на многозначные функции, напомню следующие - стандартные - определения и обозначения.

ОПРЕДЕЛЕнИЕ 1. Многообразием 1-струй $J^{1}\left(B^{n}, \mathbb{R}\right)$ функиий на $B^{n}$ назьвается многообразие $M^{2 n+1}=T^{*} B^{n} \times \mathbb{R}$ с контактной 1-формой $d z-p d q$ (где $p d q$-форма действия на фазовом пространстве $T^{*} B^{n}$, а $z$-координата на $\mathbb{R}$ ).

ПримеР. Если $B^{n}=S^{1}$ - окружность, то многообразие $J^{1}\left(B^{n}, \mathbb{R}\right)$ диффеоморфно полноторию (внутренности тора $S^{1} \times \mathbb{R}^{2}$ ). На этом многообразии имеются координаты $(q \bmod 2 \pi, p, z)$. 
ОПРЕДЕЛЕНИЕ 2. Лежандровым подмногообразием $\Lambda^{n} \subset M^{2 n+1}$ называется подмногообразие, на котором контактная 1-форма обращается в нуль.

ПРИмеР. Любой функции $g: B^{n} \rightarrow \mathbb{R}$ соответствует лежандрово сечение $\Lambda^{n}$ расслоения $J^{1}\left(B^{n}, \mathbb{R}\right) \rightarrow B^{n}$, заданное формулами

$$
p=\frac{\partial g}{\partial q}, \quad z=g(q)
$$

Многообразие $\Lambda$ зависит лишь от функции $g$, а не от выбора локальных координат: формула (1) сопоставляет точке $q$ из $B^{n}$ кокасательньй вектор $d g$ и число $g(q)$.

ОПрЕДЕЛЕниЕ 3 . Вложенное лежандрово подмногообразие $\Lambda^{n} \subset J^{1}\left(B^{n}, \mathbb{R}\right)$ называется квазифункиией на $B^{n}$, если оно принадлежит компоненте связности нулевого сечения $(p=0, z=0)$ в пространстве вложенных лежандровых подмногообразий многообразия 1-струй функций на $B^{n}$.

Проекция квазифункции из пространства 1-струй в фазовое пространство (при естественном отображении забывания значения функции) является точным лагранжевым подмногообразием в $T^{*} B^{n}$.

Это подмногообразие может оказаться не вложенньм, а лишь иммерсированным в $T^{*} B^{n}$ (имеющим самопересечения).

Всякое точное лагранжево подмногообразие $L^{n}$, иммерсированное в $T^{*} B^{n}$, получается этим способом из некоторого лежандрова многообразия $\Lambda^{n}$ в $J^{1}\left(B^{n}, \mathbb{R}\right.$ ) (определенного с точностью до сдвигов оси $z$, если $L^{n}$ связно).

Лежандрово многообразие $\Lambda^{n}$ может, однако, оказаться лишш иммерсированным (имеющим самопересечения в $2 n+1$-мерном многообразии струй $J^{1}\left(B^{n}, \mathbb{R}\right)$ ).

ПримеР. Кривая типа рис. 12 является проекцией лежандровой кривой с самопересечением, если $A=0, B=C$ (т.е. если петля, ближайшая к экватору $p=0$, точна; разумеется, эта петля должна тогда не менее двух раз пересечь экватор).

При естественной деформации нулевого сечения в кривую рис. 12 в классе точных лагранжевых иммерсий в некоторьй момент образуется именно такая ситуация самопересекающейся лежандровой кривой.

Теорема Чеканова [35], [44]-[46]. Число квазикритических точек квазифункции общего положения на $B^{n}$ (точек, где $\left.p=0\right)$ не меньше, чем сумма чисел Бетти многообразия $B^{n}$.

ПРимеР. Квазифункция на окружности имеет не менее двух квазикритических точек. Кривая рис. 12 вовсе не пересекает экватор $p=0$. По теореме Чеканова для $B^{n}=S^{1}$ эта кривая не является квазифункцией. Иньми словами, лежандровы узлы кривой рис. 12 и лежандровой кривой $(p=0, z=0)$ в пространстве $J^{1}\left(S^{1}, \mathbb{R}\right)$ различны: при деформации одной в другую в классе лежандровых кривых неизбежно возникает момент самопересечения.

Уже этот очень частный - одномерный - случай теоремы Чеканова совершенно нетривиален. Элементарное комбинаторное доказательство этого факта исчисления площадей плоских самопересекающихся кривых неизвестно.

Псевдофункции из примера $1 \S 3$ являются обобшениями квазифункций. Псевдофункция - это иммерсия окружности в $S^{2}$, а квазифункция - в цилиндр $S^{1} \times(-1,1)$. 
Отображение Архимеда (рис. 11) превращает квазифункцию в псевдофункцию. Из теоремы Чеканова следует, что так полученная псевдофункция пересекает каждую окружность большого круга сферы не менее двух раз.

Аналогичным свойством обладает и любая псевдофункция:

ТеОрема ГИвентАЛЯ [47]. Всякая псевдофункиия $S^{1}$ на сфере $S^{2}$ пересекает каждую окружность большого круга не менее двух раз.

В действительности Гивенталь доказал многомерный аналог этой теоремы, в котором роль симплектической сферы $S^{2}$ играет комплексное проективное пространство $\mathbb{C P}^{n}$, а роль лагранжевой окружности $S^{1}$ - вещественное проективное пространство $\mathbb{R P}^{n}$. Одномерное комплексное проективное пространство - это сфера Римана $\mathbb{C P}^{1} \approx S^{2}$, а вешественное - это окружность $\mathbb{R P}^{1} \approx S^{1}$.

Теорема Гивенталя показывает, что неравенства Морса сохраняют силу для многозначных функций на окружности, если понимать их как псевдофункции (а не только как квазифункции теоремы Чеканова). Можно надеяться, что и обобщение теоремы Гурвища (эта теорема соответствует теореме о теннисном мяче для точных инфинитезимальных возмушений экватора) сохраняет силу даже и для очень больших возмущений, при которых роль функции на окружности играет псевдофункция на сфере (гипотеза примера $1 \S 3$ ). Эта гипотеза, впрочем, не доказана и для частного случая квазифункций Чеканова, являющихся самопересекающимися кривыми на поверхности цилиндра.

Вероятно, простейшим вопросом этого рода является следующий. Рассмотрим $2 \pi$-периодическую по $q$ функцию $F(x, q)$, равную $x^{2}$ при больших $x$. Пусть уравнение $\partial F / \partial x=0$ определяет на цилиндре $\{(x, q \bmod 2 \pi)\}$ вложенную гладкую кривую $\gamma$, гомотопную экватору $x=0$.

Если $F$ не слишком сильно отличается от $x^{2}$, то функция $p(q)=\partial F /\left.\partial q\right|_{\gamma}$ однозначна. В этом случае уравнение $\partial^{2} p / \partial q^{2}+p=0$ имеет не менее четырех решений. Неизвестно, остается ли этот результат в силе, когда функции $x(q)$ и $p(q)$ многозначны?

ЗАмЕчАнИЕ. В отличие от теоремы о теннисном мяче, в большинстве других примеров топологических оценок числа особых точек снизу естественные ограничения (скажем, в терминах лежандровых узлов), аналогичные понятию псевдофункции, еще не сформулированы даже и в виде гипотез.

\section{§ 7. Лагранжев коллапс и точки возврата каустик}

В терминах симплектической геометрии теорема о четырех точках возврата каустики (применимая как к геодезическим на близких к сфере поверхностях, так и к вершинам плоских кривых и огибаюшим ларморовских окружностей) состоит в следующем.

Рассмотрим лагранжево многообразиев фазовом пространстве $T^{*} \mathbb{R}^{n}$, соответствуюшее (двузначной) функции $z= \pm|q|$.

Это многообразие $L^{n}$ состоит из векторов $p= \pm q /|q|$ длины 1 , приложенных к каждой точке $q$ (направленных как радиус-вектор $q$ или строго противоположно) и из всех векторов длины 1, приложенных в нуле. 
ЛЕмма. Множество $L^{n}$ представляет собой гладкое многообразие, диффеоморфное иилиндру $S^{n-1} \times \mathbb{R}$, и является точнымм лагранжсевым подмногообразием фазового пространства.

ДокаЗАТЕльство. Диффеоморфизм $S^{n-1} \times \mathbb{R} \rightarrow L$ задается формулами $(p, t) \mapsto$ $(q=p t, p)$. Поэтому на $L p d q=p^{2} d t=d t$. Значит, $L$ точно и $t$ - соответствующий потенциал.

ОПРЕДЕЛЕНИЕ. Лагранжсевым отображсением называется проектирование лагранжева подмногообразия фазового пространства на конфигурационное многообразие. Каустикой лагранжева отображения называется множество его критических значений.

Каустика лагранжева отображения общего положения - это гиперповерхность с особенностями в конфигурационном многообразии.

ПримеР. Каустика лагранжева отображения на двумерную поверхность общего положения - кривая, особенности которой - точки возврата полукубического типа и точки трансверсального самопересечения.

Лагранжево подмногообразие $L^{n} \subset T^{*} \mathbb{R}^{n}$ предыдушей леммы бесконечно вырожденно: его каустика представляет собой одну точку (прообразом которой является целая сфера критических точек $\left.S^{n-1}\right)$.

ОПрЕДЕЛЕниЕ. Бесконечно-вырожденное лагранжево отображение проектирования $L^{n} \rightarrow \mathbb{R}^{n}$ назьвается стандартным лагранжевым коллапсом.

Стандартный лагранжев коллапс напоминает сжатие (антисигмапроцесс, blowing down) алгебраической геометрии: это отображение является локальным диффеоморфизмом, двулистно накрьвающим $\mathbb{R}^{n} \backslash\{0\}$, а в начало координат коллапсирует целая cфера.

ПримеР. Рассмотрим на стандартной евклидовой сфере $S^{n}$ геодезические, выходящие со скоростью 1 из северного полюса. Отождествим их векторы скорости с кокасательными векторами сферы (при помощи ее римановой метрики). Мы получим лагранжево многообразие $L^{n}$ в фазовом пространстве $T^{*} S^{n}$.

ЛЕмма. Лагранжево отображение $L^{n} \rightarrow S^{n}$ имеет над каждым из полюсов особенность стандартного лагранжева коллапса.

ЗАмЕчАниЕ. Эквивалентность лагранжевых проектирований - это симплектоморфизм пространств расслоений, переводящий первое проектируемое лагранжево подмногообразие во второе и слои первого расслоения - в слои второго.

ДокаЗАТЕльство. Рассмотрим функции "расстояние до полюса" на $S^{n}$ и "расстояние до начала координат" в $\mathbb{R}^{n}$. Эти две функции локально диффеоморфны (так как их квадраты диффеоморфны по лемме Морса). Этот диффеоморфизм и индуцирует эквивалентность обоих лагранжевых отображений.

Рассмотрим теперь точное двумерное лагранжево многообразие общего положения, близкое (вместе с производными) к многообразию $L$ стандартного лагранжева коллапса. 
ТЕОрема [5]. Каустика возмущенного лагранжева отображения имеет не менее четырех точек возврата.

ПримеР. Возмутим евклидову метрику двумерной сферы. Геодезические, выходящие из северного полюса сферы, по-прежнему образуют в фазовом пространстве лагранжево точное многообразие над достаточно малой окрестностью южного полюca.

Полученное лагранжево проектирование является возмушением исходного. По теореме каустика имеет не менее четырех точек возврата.

Это же рассуждение применимо ко второй каустике (вблизи северного полюса), третьей (вблизи южного) и т. д. (но, разумеется, возмушение метрики должно быть тем меньшим, чем вьше номер каустики, чтобы возмущение лагранжева многообразия оставалось малым).

ЗАмЕчАнИЕ 1. Неясно, насколько малым должны быть возмушения лагранжева цилиндра. Например, в частном случае задачи Якоби о геодезических на сфере, достаточна положительность кривизны возмущенной метрики, а в задаче о четырех вершинах - выпуклость кривой. Условия эти можно, вероятно, значительно ослабить (в духе теоремы Чеканова из $\S 6$ ).

ЗАМЕчАНИЕ 2. В многомерном случае вопрос о топологически необходимых особенностях маловозмущенного стандартного лагранжева коллапса не решен.

ГИПоТЕЗА (В.М. Закалюкин). Минимальный набор особенностей мальх возмущений стандартного лагранжсева коллапса реализуется нормальным отображсением әллипсоида в евклидовом пространстве.

Нормальное отображение подмногообразия (например, гиперповерхности) в евклидовом пространстве определяется следуюшим образом. Отождествим касательные векторы с кокасательными при помощи евклидовой метрики. Все векторы, нормальные подмногообразию, определяют (точное) лагранжево подмногообразие $L_{0}$ в фазовом пространстве $T^{*} \mathbb{R}^{n}$. Рассмотрим образ $L$ этого подмногообразия $L_{0}$ под действием преобразования за время 1 в геодезическом потоке евклидова пространства: $L=g^{1} L_{0}$.

ОПреДЕЛЕниЕ. Лагранжево проектирование $L^{n} \rightarrow \mathbb{R}^{n}$ называется нормальным отображением для исходного подмногообразия.

ПРИмЕР. Нормальное отображение для кривой на плоскости - это отображение цилиндра, образованного всеми нормальными кривой векторами, на плоскость, сопоставляющее каждому вектору его конец.

Каустикой нормального отображения для гиперповерхности является ее фокальное множество. Гипотеза Закалюкина утверждает, что фокальное множество эллипсоида реализует минимальный набор топологически необходимых особенностей всевозможных лагранжевых проектирований, достаточно близких к стандартному лагранжеву коллапсу.

Например, фокальная кривая эллипса имеет четыре точки возврата. В этом случае гипотеза доказана. (В этом и состоит предыдушая теорема, доказываемая при помощи теоремы Гурвица типа Штурма о рядах Фурье.) 
Фокальная поверхность эллипсоида в трехмерном пространстве имеет три ребра возврата.

Закалюкин [4] получил первые результаты о топологически необходимых особенностях возмущений лагранжева коллапса в размерности $n>2$. Но, например, необходимы ли 3 ребра возврата, остается, кажется, неизвестным.

ЗАмЕчАниЕ 3. Лагранжев цилиндр стандартного лагранжева коллапса можно продеформировать так, чтобы сохранить топологические свойства его бесконечно-вырожденного лагранжева проектирования.

А именно, рассмотрим произвольный лагранжев цилиндр $L^{n} \approx S^{n-1} \times \mathbb{R}$ в $T^{*} \mathbb{R}^{n}$, пересечение которого со слоем $T_{0}^{*} \mathbb{R}^{n}$ представляет собой (квадратично) выпуклую гиперповерхность в этом евклидовом пространстве (причем касательные пространства цилиндра и слоя нигде не совпадают).

Теорема о четырех точках возврата каустики возмущения лагранжева коллапса в этой ситуации доказана М.Э. Казаряном [48].

\section{§ 8. Лежандров коллапс и точки возврата фронтов}

Теорема о четырех точках возврата выворачивающегося наизнанку волнового фронта на плоскости также допускает абстрактную формулировку в терминах лежандрова коллапса в контактной геометрии.

Рассмотрим многообразие коориентированных контактных элементов $S T^{*} B^{n}$ многообразия $B^{n}$. Точкой этого $(2 n-1)$-мерного многообразия (контактнылм әлементом на $B$ ) является коориентированная гиперплоскость в касательном пространстве к $B$ в какой-либо точке. Фиксировав на $B$ какую-либо риманову метрику, мы можем задавать коориентированные контактные элементы ортогональными им векторами длины 1.

Естественная контактная структура (невырожденное поле касательных гиперплоскостей) многообразия $M^{2 n-1}$ контактных элементов на $B^{n}$ задается следующим "условием конька": контактный элемент имеет право врашаться и двигаться в принадлежащем ему направлении, но не в поперечном направлении. Инњми словами, естественная контактная структура на $M$ - это “тавтологическое" распределение гиперплоскостей: точка из $M$ является гиперплоскостью в касательном пространстве к $B$, прообраз этой-то гиперплоскости при естественной проекции $M \rightarrow B$ и есть гиперплоскость тавтологического распределения в касательном пространстве к $M$.

ОПРЕДЕЛЕНИЕ. Подмногообразие многообразия контактных элементов называется лежсандровым, если оно является интегральным многообразием максимальной возможной размерности естественной контактной структуры на $M$.

Размерность лежандровых подмногообразий в $M^{2 n-1}=S T^{*} B^{n}$ равна $n-1$.

Пример 1. Все контактные элементы на $B$, касающиеся одной гиперповерхности $X^{n-1} \subset B^{n}$, образуют лежандрово подмногообразие в $M$.

ПримеР 2. Для гладкого подмногообразия $X^{k} \subset B^{n}$ любой размерности (даже размерности $k=0)$ многообразие всех $\left(n-1\right.$-мерных) контактных элементов на $B^{n}$, касаюшихся $X$, лежандрово. 
ОПРЕДЕЛЕНИЕ. Фронтом лежандрова подмногообразия $\Lambda^{n-1} \subset S T^{*} B^{n}$ называется проекция $\Lambda$ в $B$.

Фронт лежандрова подмногообразия общего положения является гиперповерхностью с особенностями в $B$.

ПримеР. Фронты лежандровых кривых обшего положения на плоскости $B=\mathbb{R}^{2}$ это кривые с полукубическими точками возврата (возможно, самопересекающиеся).

ОПРЕДЕЛЕНИЕ. Стандартным лежандровым коллапсом в $\mathbb{R}^{n}$ называется лежандрово проектирование из $S T^{*} \mathbb{R}^{n}$ в $\mathbb{R}^{n}$ однопараметрического семейства лежандровых многообразий, образованных коориентированными касательньми плоскостями сфер радиуса $t \in \mathbb{R}$ с центром в точке $0 \subset \mathbb{R}$ (при $t>0$ коориентируюший вектор направлен наружу, при $t<0$ внутрь сферы; значению параметра $t=0$ соответствует сфера, образованная всеми контактньми элементами, приложенными в 0).

ПРИмЕР. При $n=2$ стандартный лежандров коллапс определяет проектирование однопараметрического семейства лежандровых окружностей на плоскость. Фронты, соответствуюшие $t \neq 0$, являются окружностями на плоскости. Соответствующая значению параметра $t=0$ лежандрова окружность целиком отображается в одну точку. Этот фронт бесконечно вырожден.

При малом финитном (сосредоточенном в конечной области) возмушении семейства (в классе однопараметрических семейств лежандровых кривых) получится новое семейство, фронты которого при $t>c$ - замкнутые несамопересекаюшиеся кривые, коориентированные направлением "наружу", а при $t<-c-$ внутрь.

Промежуточньм значениям параметра $t$ отвечает "выворачивание" фронта. Для $t$ обшего положения фронт - кривая с точками возврата и с точками самопересечения (при некоторых особых значениях $t$ происходят еще и перестройки, в том числе перестройки рождения или уничтожения пар точек возврата фронта).

Teорема (См. [8], [9], [10]). Среди промежуточных фронтов всегда имеются фронты с не менее чем четырьмя точками возврата.

Для инфинитезимальных возмущений стандартного лежандрова коллапса этот результат сводится (после некоторых выгислений) к теореме Гурвица типа Штурма. Теорема утверждает, что он остается справедливьм и для конечных возмущений.

ЗАМЕчАНИЕ 1. В действительности, доказательство показьвает, что результат справедлив даже и для весьма больших возмушений (оценка их величины приведена в [8]). Гипотетически он остается верньм и для очень больших возмушений. Важным ограничением является отсутствие самопересечений на всех лежандровых узлах в трехмерном многообразии $S T^{*} \mathbb{R}^{2} \approx S^{1} \times \mathbb{R}^{2}$, составляющих продеформированное семейство лежандровых кривых (или даже составляющих все семейства, интерполируюшие стандартное семейство с продеформированным). Не исключено, что это единственное нужное здесь ограничение (подобно ситуации в теореме Чеканова).

ЗАмечАниЕ 2. Теории Закалюкина и Казаряна (см. $\S 7$ ) несомненно могут быть переформулированы и в терминах лежандровых коллапсов, но это, сколько мне известно, пока не сделано. 


\section{§9. Пространственные кривые и их точки уплощения}

Все предыдущие теоремы о четырех особенностях были обобщениями случая $N=2$ теоремы Гурвища типа Штурма. Случай произвольного $N$ (соответствующий $2 N$ особенностям) возникает при исследовании точек уплощения пространственных кривых.

Гладкое отображение обшего положения $f: S^{1} \rightarrow \mathbb{R}^{m}$ является (при $m>2$ ) вложением. Более того, для такого отображения производные порядков $(1, \ldots, m-1)$ в каждой точке линейно независимы. Гиперплоскость, натянутая на эти векторы в точке кривой, назьвается соприкасающейся.

Производная порядка $m$ в точке общего положения не лежит в соприкасаюшейся гиперплоскости. Однако для кривой общего положения в отдельных точках эта производная попадает в соприкасающуюся гиперплоскость. Такие точки называются точками уплощения.

ПРИмЕР. Точки уплощения плоских кривых - это точки перегиба (где обращается в нуль кривизна). Точки уплошения кривых в $\mathbb{R}^{3}$ - это точки, где обрашается в нуль кручение. Для кривых обшего положения эти нули - первого порядка.

Понятие точки уплошения - проективное, так как уплощение - это пересечение необычно высокой кратности с соприкасающейся гиперплоскостью. Поэтому точки уплощения определены и для кривых в проективных пространствах.

Пространственная кривая в $\mathbb{R}^{3}$ имеет не менее четырех точек уплощения, если она близка (с производньми) к выпуклой плоской кривой (пример $3 \S 3$ ). Близость с производными здесь и далее обязательна, так как спираль, лежашая на поверхности тонкого тора, осью которого является плоская выпуклая кривая, вовсе не имеет точек уплощения.

Чтобы сформулировать обобщение теоремы о четырех точках уплощения на кривые в (проективных) пространствах бо́льшего числа измерений, начнем с обобшения понятия выпуклости (следуя [27]).

ОПРЕДЕЛЕНИЕ. Вложение окружности в проективное пространство $\mathbb{R} \mathrm{P}^{m}$ называется выпуклой кривой, если число точек пересечения образа с любой гиперплоскостью не превосходит $m$ (с учетом кратностей).

ЗАмЕчАниЕ. Меньшее $m$ число взять нельзя, так как через любые $m$ точек кривой проходит гиперплоскость в $\mathbb{R P}^{m}$.

ПримеР 1. Плоская замкнутая кривая в $\mathbb{R}^{2}$ выпукла в смысле этого определения, если она является границей выпуклой области и имеет везде строго положительную кривизну.

ПримеР 2. Кривая (“обобщенный эллипс") в $\mathbb{R}^{2 n}$, заданная уравнениями

$$
x_{1}=\cos t, y_{1}=\sin t, \ldots, x_{n}=\cos n t, y_{n}=\sin n t(t \bmod 2 \pi)
$$

вьпукла. Действительно, точки ее пересечения с гиперплоскостью определяются из уравнений $x^{2}+y^{2}=1, P(x, y)=0$, где $P$ - многочлен степени $n$. По теореме Безу их число (с учетом кратностей) не превосходит $2 n$.

Выпуклая кривая не имеет точек уплощения. 
Tеорема (С. Анисов [50], М. Шапиро [49]). Всякая выпуклая кривая в четномерном пространстве $\mathbb{R P}^{2 n}$ не пересекает некоторую гиперплоскость. Такая кривая стягиваема и может бить реализована в евклидовом пространcmвe $\mathbb{R}^{2 n}$.

Выпуклая кривая в нечетномерном пространстве $\mathbb{R P}^{2 n-1}$ пересекает некоторую гиперплоскость в одной точке (под ненулевым углом). Такая кривая нестягиваема и не может быть реализована в евклидовом пространстве $\mathbb{R}^{2 n-1}$.

ПримеР. Кривая в $\mathbb{R P}^{2 n-1}$, заданная однородными координатами

$$
x_{1}=\cos t, y_{1}=\sin t, \ldots, x_{n}=\cos (2 n-1) t, y_{n}=\sin (2 n-1) t(t \bmod \pi)
$$

вьпукла.

ЗАмЕЧАнИЕ. Согласно М. Шапиро [50], выпуклые кривые в $\mathbb{R} \mathrm{P}^{2 n}$ образуют связное множество в пространстве вложений $S^{1} \rightarrow \mathbb{R} \mathrm{P}^{2 n}$, а пространство выпуклых кривых в $\mathbb{R} \mathrm{P}^{2 n-1}$ имеет две связные компоненты в пространстве вложений (репер, образованньй производными, ориентирует нечетномерное проективное пространство).

Теперь мы можем сформулировать многомерную теорему об уплощениях.

Теорема [27]. Рассмотрим малое пространственное возмущение выпуклой кривой, лежсащей в гиперплоскости проективного пространства $\mathbb{R P}^{m}$.

Возмущенная кривая имеет не менее, чем $m+1$ точку уплощения.

Доказательство (имеюшееся в [27]) сводит теорему к теореме Гурвица типа Штурма для возмущений обобшенного эллипса и к ее обобщению - для общего случая.

ПримеР 1. Лежащее в $\mathbb{R}^{5}$ достаточно малое возмущение обобщенного эллипса в $\mathbb{R}^{4}$ имеет не менее шести точек уплощения.
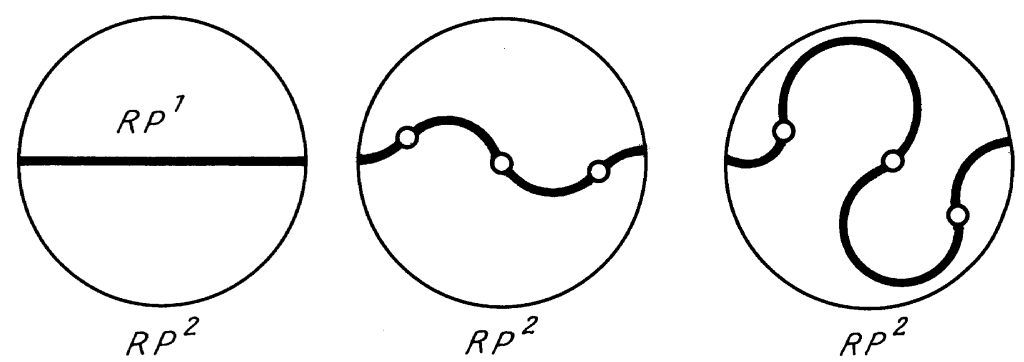

Рис. 13. Точки перегиба возмушения проективной прямой

ПримеР 2 (теорема Мёбиуса). Рассмотрим проективную прямую на проективной плоскости (рис. 13).

ТЕОремА. Достаточно близкая $к$ проективной прямой замкнутая гладкая кривая имеет не менее трех точек перегиба.

Этот результат, по-видимому, принадлежит Мёбиусу и тесно связан с открытием ленты Мёбиуса, из неориентируемости которой следует, что число точек перегиба всегда нечетно. 
В действительности возмушения здесь могут быть весьма большими. Достаточно, например, чтобы продеформированная кривая оставалась нестягиваемой и несамопересекающейся (подробности см. в [51]).

Если самопересечения допускаются, то трех точек перегиба может и не быть (рис. 14), даже если иммерсированная кривая регулярно гомотопна вложенной прямой.
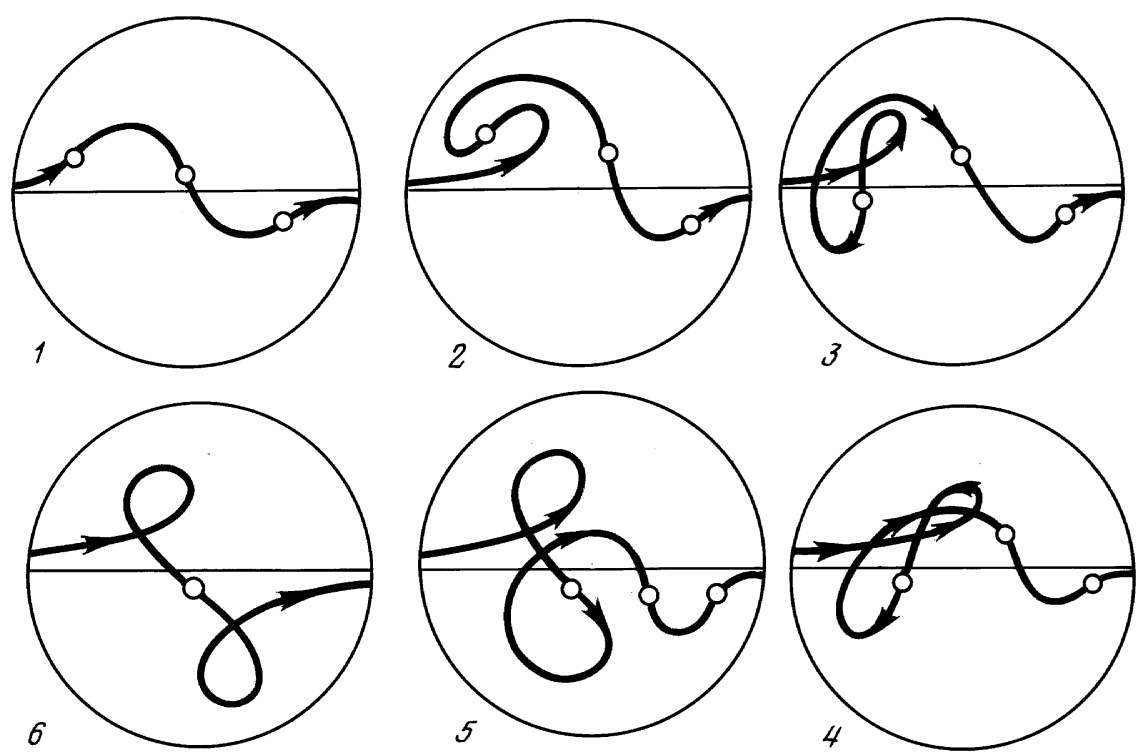

Рис. 14. Исчезновение пары точек перегиба при деформации иммерсии

ГИПОТЕЗА. Три точки перегиба на иммерсированной кривой сохраняются до тех пор, пока при деформации не произойдет самокасания одинаково ориентированных ветвей (т.е. пока соответствующий лежсандров узел не перестроuтся).

Недавно Д. Панов доказал эту гипотезу при дополнительном предположении, что ни одна их участвуюших в регулярной гомотопии кривых не имеет более пяти точек перегиба.

ЗАМЕчАНИЕ. Двойственная к исходной прямой кривая состоит из одной точки. Двойственная к возмушенной кривой кривая является фронтом лежандровой кривой (лежашей в пространстве неориентированных контактных элементов проективной плоскости $\left.P T^{*} \mathbb{R P}^{2}\right)$.

Теорема Мёбиуса о трех точках перегиба утверждает, что при возмущении точечного фронта возникает фронт с не менее чем тремя точками возврата.

Эта теорема является неориентированным вариантом теоремы о лежандровом коллапсе. В случае неориентированных контактных элементов топологически необходимые особенности должны присутствовать на фронте любого малого индивидуально- 
го лежандрова возмушения слоя проективизированного кокасательного расслоения $P T^{*} \mathbb{R}^{2} \rightarrow \mathbb{R}^{2}$

При каких естественных ограничениях на возмущения кривых в $\mathbb{R P}^{m}$ сохраняет силу теорема о точках уплощения при бо́льших значениях $m$ - неизвестно (не сформулированы даже гипотезы типа теоремы Чеканова).

\section{§ 10. Вершины выпукльх пространственных кривых}

В качестве следствия теоремы о точках уплощения пространственной кривой в нечетномерном евклидовом пространстве можно получить следующее многомерное обобшение теоремы о четырех вершинах для выпуклых кривых в пространстве предыдущей четной размерности.

ОПРЕДЕЛЕНИЕ. Вершиной кривой в $\mathbb{R}^{m}$ называется стационарная точка кривизны соприкасаюшейся сферы.

( Соприкасающейся называется сфера, к которой кривая всего ближе в данной точке, пересекая ее с кратностью, бо́льшей чем $m$. В вершине эта кратность еше больше.)

ЗАмЕчАнИЕ. При $m=2$ вершины - это стационарные точки кривизны кривой. При $m>2$ кривизна соприкасаюшейся сферы зависит не только от кривизны и кручений кривой, но и от их производных.

Теорема (Р. Урибе [52]). Выпуклая кривая в $\mathbb{R}^{2 n}$ имеет не менее $2 n+2$ вериин.

Доказательство основано на следующей идее (см. работу В. Д. Седых [53], где из этой идеи извлекается другая информация). Подходящая стереографическая проекция $\mathbb{R}^{2 n}$ на сферу $S^{2 n}$ большого радиуса в $\mathbb{R}^{2 n+1}$ делает нашу вьпуклую кривую пространственным возмущением выпуклой кривой, лежашим в $\mathbb{R}^{2 n+1}$. Эта пространственная кривая имеет не менее $2 n+2$ точек уплошения.

Лемма. Вершины исходной кривой переходят при стереографической проекции в точки уплощения пространственной кривой.

ДокАЗАтЕльство. Соприкасаюшаяся плоскость пространственной кривой пересекает сферу большого радиуса $S^{2 n}$ по сфере $S^{2 n-1}$. Прообраз этой $(2 n-1)$-мерной сферы при стереографической проекции $\mathbb{R}^{2 n}$ на $S^{2 n}$ тоже является $(2 n-1)$-мерной сферой. Эта гиперсфера в $\mathbb{R}^{2 n}$ имеет необычно высокую кратность пересечения с кривой в $\mathbb{R}^{2 n}$, если и только если кривая-образ в $\mathbb{R}^{2 n+1}$ пересекает свою соприкасающуюся гиперплоскость с необычно высокой кратностью. Лемма доказана.

По лемме из существования $2 n+2$ точек уплошения у возмущенной кривой в $\mathbb{R}^{2 n+1}$ вытекает сушествование $2 n+2$ вершин у невозмущенной кривой в $\mathbb{R}^{2 n}$.

ЗАмЕЧАНИЕ. В теории выпуклых пространственных кривых (известной также под названием теории чебьшевских систем функций на окружности) много интересных фактов и открытых вопросов. Например: всякая ли выпуклая кривая в $\mathbb{R}^{2 n}$ имеет вьпуклую проекцию в $\mathbb{R}^{2 n-2}$ ? Является проекцией выпуклой кривой из $\mathbb{R}^{2 n+2}$ ?

Б. Хесин и В. Овсиенко, а также М. Казарян доказали, что кривая, проективно двойственная выпуклой, выпукла (см. [54], [27]), причем переход к проективно двойственной вьпуклой кривой соответствует на языке дифференциальных операторов переходу к оператору, двойственному по Лагранжу. 
Рассмотрим вьпуклую кривую в $\mathbb{R} \mathrm{P}^{2 n}$. Плоскость $\mathbb{R} \mathrm{P}^{2 n-2}$ называется внутренней по отношению к кривой, если каждая гиперплоскость, проходяшая через нее, пересекает кривую в $2 n$ точках. Коэффищиент зацепления $\mathbb{R}^{2 n-2}$ с кривой в $\mathbb{R}^{2 n}$ в этом случае равен $n$.

ПРИМЕР. При $n=1$ внутренние точки - это точки выпуклой области, ограниченной кривой на плоскости.

Сушествование внутренних точек для многомерных выпуклых кривых недавно независимо доказано С. Анисовым и С. Гусейн-Заде, исходя из топологических соображений.

Прямая $\mathbb{R P}^{1}$ назьвается внешней по отношению к выпуклой кривой в $\mathbb{R} \mathrm{P}^{2 n}$, если через каждую точку этой прямой проходит $2 n$ соприкасающихся гиперплоскостей кривой.

ПримеР. При $n=1$ внешние прямые - это все прямые, не пересекаюшие кривую на плоскости. Существование внешних прямых следует из существования внутренних точек для двойственной кривой.

Соприкасающаяся гиперплоскость выпуклой кривой в точке более ее не пересекает (так как кратность пересечения в одной этой точке уже достигает максимального возможного значения).

Для кривых на плоскости условие отсутствия лишних точек пересечения с соприкасаюшейся гиперплоскостью (т.е. с прямой) эквивалентно выпуклости. Сохраняется ли эта эквивалентность для пространственных кривых - неизвестно: верно ли, что кривая, имеющая с каждой своей соприкасающейся гиперплоскостью ровно одну общую точку (кратности, равной размерности пространства) - выпуклая?

Tеорема ([30]: уточнение гипотезы М. Казаряна). Проекиия вылуклой кривой, лежащей в $\mathbb{R P} \mathrm{P}^{m}$, из не лежащего на кривой центра в пространство $\mathbb{R P}^{m-1}$ имеет не более $m$ точек уплощения, причем существуют проекции, имеющие ровно $m, m-2, m-4, \ldots$ точек уплощения.

ДокАЗАТЕЛЬСтвО. Точки уплощения проекции получаются из тех точек кривой, для которых соприкасающаяся плоскость содержит центр проектирования. Двойственная центру гиперплоскость пересекает двойственную исходной кривой кривую по точкам, двойственньм этим соприкасающимся плоскостям. Поскольку кривая, двойственная выпуклой, выпукла, число этих точек пересечения не превосходит $m$. Через любые $m$ точек на двойственной кривой проходит гиперплоскость. Проекция из двойственного этой гиперплоскости центра имеет ровно $m$ точек уплощения.

ЗАмЕчАниЕ. Каждая выпуклая кривая (например, обобщенньй эллипс) задает стратификацию двойственного проективного пространства (в соответствии с кратностями пересечений гиперплоскостей с кривой).

Эта стратификация мало изучена. Например, верно ли, что она топологически (и комбинаторно) одинакова для всех выпуклых кривых в проективном пространстве данной размерности?

Даже в случае обобщенного эллипса нет хорошего описания компонент связности многообразия гиперплоскостей общего положения (дополнения к дискриминантной 
гиперповерхности в двойственном проективном пространстве, образованной не трансверсальными кривой гиперплоскостями). Неизвестно даже число этих компонент.

Можно предполагать, что все эти вопросы допускают достаточно простые ответы (вероятно, в терминах зеркал аффинных групп Вейля).

Близкая задача о топологической классификации вешественшых тригонометрических многочленов также остается открытой (она соответствует стратификации линейных функций в $\mathbb{R}^{2 n}$ в зависимости от критических точек их ограничений на обобщенный эллипс).

Верно ли, что числа компонент связности пространства вещественных тригонометрических многочленов степени $n$ с 2 р различными критическими значениями образуют последовательность чисел Эйлера $1,5,61, \ldots$ ?

Это верно для пространства всех периодических функций. См. [55], где также показано, что числа тангенса (образующие вместе с числами Эйлера последовательность $1,1,2,5,16,61, \ldots$ с экспоненциальной производящей функцией $\sum K_{n} t^{n} / n !=\sec t+$ $\operatorname{tg} t)$ считают компоненты пространств мёбиусовских функций общего положения на окружности.

Вопрос о топологической классификации вещественных тригонометрических многочленов тесно связан с вопросом об отображении Ляшко-Лойенги для этого случая, с топологической классификацией комплексных рациональных функций и с формулой Кэли для числа деревьев с нумерованными ребрами (см. [56]-[63]). Тригонометрическим многочленам соответствуют рациональные функции с двумя полюсами и графы с одним циклом.

Будем называть $n$-графом связньй граф с $n$ ребрами и $n$ вершинами, у которого обе вершины каждого ребра различны.

ТЕорема. Топологические типь рациональных функций общего положения с двумя полюсами суммарной кратности $n$ находятся во взаимнооднозначном соответствии с п-графами с нумерованными ребрами.

ПримеР. Существуют два 3-графа: $\Delta$ и •-•-•. Первый имеет единственную нумерацию, а второй - три различных нумерации ребер. Итого четыре нумерации.

В соответствии с этим имеются четыре топологически различных рациональных функции с двумя полюсами суммарной кратности 3 , с фиксированными критическими значениями. Под топологической эквивалентностью функций здесь понимается гомеоморфизм $h$ пространства прообраза: $f \sim g$, если $f(z) \equiv g(h(z))$.

ДокАЗАТЕЛЬСтво ТЕОРЕмЫ. Соответствие между функциями и $n$-графами строится следуюшим образом (эта конструкция в полиномиальной ситуации принадлежит С. Здравковске [58]). Рассмотрим $n$ критических значений $c_{1}, \ldots, c_{n}$ рациональной функции. Разобьем сферу значений на два гладких “диска” с “центрами” * и $\infty$, на обшей окружности которых лежат критические значения.

Точка $*$ - регулярное значение. Она имеет $n$ прообразов. Эти точки будут вершинами нашего $n$-графа. Соединим базисную точку $*$ с критическим значением $c_{i}$ “радиусом” диска. При движении значения к критическому две точки прообраза сливаются, образуя исчезающий цикл критической точки. Соответствующие две точки прообраза базисной точки * соединены в $n$-графе $i$-м ребром. Мы получили $n$-граф с нумерованньми ребрами (зависящий от выбора дисков и от нумерации критических значений). 
Обратно, по $n$-графу с нумерованными ребрами (при фиксированных дисках и нумерованных критических значениях) можно восстановить рациональную функцию (с точностью до умножения аргумента на константу и преобразования $z \rightarrow 1 / z$, меняюшего полюса местами).

Для этого разрежем сферу-образ по “радиусам” внешнего “диска”, соединяющим критические значения с точкой $\infty$, и склеим риманову поверхность из $n$ экземпляров взрезанной сферы значений, отождествляя противоположные берега $i$-го разреза на листах, соответствуюших концам $i$-го ребра $n$-графа.

ЛЕмМА. Полученная в результате склейки поверхность является сферой. Число прообразов точки $\propto$ на ней равно 2.

ДокАЗАТЕльство. Это следует из того, что сфера - единственная ориентируемая связная поверхность с положительной эйлеровой характеристикой. Обозначим число прообразов точки $\infty$ через $k$. Рассмотрим на сфере-образе $n$ ребер, соединяющих критические значения с точкой $\infty, n+1$ вершину (точка $\infty$ и критические значения) и область, остающуюся после разрезания.

Полньй прообраз на построенной поверхности содержит $n^{2}$ ребер, $k+n(n-1)$ вершин и $n$ областей. Эйлерова характеристика построенной поверхности равна, таким образом, $k$. Но $k$ положительно, а эйлерова характеристика поверхности рода $g$ равна $2-2 g$. Следовательно, $k=2$, что и требовалось доказать.

ЗАмЕчАнИЕ. Мы получили, между прочим, топологическое доказательство чисто комбинаторного факта: произведение $n$ транспозиций множсества из $n$ әлементов, заданное п-графом, состоит (при любом порядке сомножителей) из ровно двух ииклов.

Комплексная структура на построенной поверхности индуцируется комплексной структурой сферы-образа. По теореме Римана она стандартна: поверхность-прообраз является сферой Римана с комплексной координатой $z$. Ее естественное отображение на сферу-образ голоморфно. Поэтому оно задается рациональной функцией от $z$. Число прообразов точки $\infty$ по лемме равно 2. Поэтому эта рациональная функция имеет ровно два полюса.

Выбирая на сфере-прообразе координату $z$, определенную лишь с точностью до дробно-линейного автоморфизма, мы можем перевести эти две точки-полюсы в $z=0$ и $z=\infty$. Этими условиями координата $z$ (а тем самьм и рациональная функция) определяется с точностью до возможности умножить $z$ на константу или поменять 0 и $\infty$ местами (эти операции не меняют ни критические значения, ни топологический тип отображения).

ЗАмЕчАниЕ. Числа $N(n) n$-графов с нумерованными ребрами даются при малых $n$ следующей таблищей.

\begin{tabular}{|c|c|c|c|c|c|}
\hline$n$ & 2 & 3 & 4 & 5 & 6 \\
\hline$N(n)$ & 1 & 4 & $39=27+12$ & $472=256+216$ & 6900 \\
\hline
\end{tabular}

Здесь указаны также числа $N(p, q)$ типов функций с полюсами порядков $p \geqslant q$, $p+q=n$ (т.е. числа $n$-графов, с нумерованньми ребрами, для которых произве- 
дение соответствующих ребрам транспозиций в порядке, соответствующем нумерации ребер, имеет два цикла порядков $p$ и $q$ ). Например, $N(1,1)=1, N(2,1)=4$, $N(3,1)=27, N(4,1)=256$.

TeOpema [70].

$N(p, q)=\frac{(p+q-1) !}{p ! q !} p^{p} q^{q} \quad$ npu $\quad p \neq q, \quad N(p, p)=\frac{(2 p-1) !}{2(p !)^{2}} p^{2 p} \quad$ npu $\quad p>1$.

ПримеР. $N(p, 1)=p^{p}, N(p, 2)=2(p+1) p^{p}$ при $p \neq 2$.

Эта теорема является своеобразным обобщением теоремы Кели о нумерованных деревьях и может быть доказана подсчетом кратности соответствуюшего отображения типа Ляшко-Лоенги, аналогичным вычислениям в [56], [57], [68], [69].

ЗАмЕчАниЕ. Вся эта комбинаторика приобретает более геометрический вид в следующей интерпретации С. Здравковски [58] (предвосхитившей “детские рисунки” Гротендика).

Расположим наш $n$-граф на плоскости так, чтобы циклический порядок ребер, выходящих из каждой вершины (заданньй ориентацией плоскости) совпадал с циклическим порядком соответствующих циклических значений на окружности “диска” с центром * (ориентированной как край этого комплексного диска).

Полученньй плоский граф̆ делит плоскость на две части, которые и соответствуют двум полюсам нашей рациональной функции. (Здравковски вместо графа рассматривает полньй прообраз “диска" с центром *, который ретрагируется на наш граф.)

Кратность каждого полюса можно следуюшим образом вычислить по построенному плоскому графу с нумерованными ребрами. Рассмотрим одну из областей, на которые граф делит плоскость. Граница этой области состоит из ребер графа. Некоторые ребра входят в нее два раза. Например, ведущее в концевую вершину ребро входит в границу соответствуюшей области два раза подряд. Мы получаем в качестве границы циклически упорядоченную последовательность ребер (быть может, с повторениями).

Определим угол между двумя последовательными ребрами следующим образом. Пусть предыдущее ребро соответствует критическому значению $a$, а последующее критическому значению $b$. Дуга с началом $a$ и концом $b$ на ориентированной окружности "диска" с центром * разбивается критическими значениями $c_{i}$ на некоторое число $r$ дуг. Это число мы и назовем углом нашей области в точке соединения предыдущего и последующего ребер. Число $r$ положительно и не превосходит $n$ (оно равно $n$ только если предыдущее и последующее ребра совпадают). Легко проверяется

ПРЕДЛОЖЕНИЕ. Кратность полюса, соответствующего данной области, равна сумме углов этой области, поделенной на $n$.

ПримеР. Граф $\square$ имеет три нумерации (0123), (0132), (0213). Соответствующие суммы углов (для той из областей, граница которой состоит из идущих в этом порядке ребер) равны, соответственно, $1+1+1+1=4,1+2+3+2=8,2+3+2+1=8$. Для дополнительной области суммы углов есть $12,8,8$. Кратности полюсов $(3,1),(2,2)$ и $(2,2)$, соответственно. Поэтому граф $\square$ вносит в $N(3,1)$ вклад 1 , а в $N(2,2)$ вклад 2 . 
Наши результаты можно интерпретировать как (нетривиальную) информацию о плоских $n$-графрах.

Развитая выше теория может быть применена и к рациональным функциям с $k>2$ полюсами (которые при $k>3$ уже нельзя фиксировать). Это приводит к графам с $n$ вершинами и $d>n$ ребрами.

Однако рациональным функциям соответствуют при $d>n$ не все нумерации ребер графа, а лишш некоторые. Действительно, пусть число циклов произведения транспозиций, соответствующего нумерации, равно $K$. Тогда эйлерова характеристика прообраза равна $K+n-d$, что может при $d>n$ оказаться не равным 2 .

Пример. Пусть $n=3, d=4$. Имеется три связных графа с тремя вершинами и четырьмя ребрами. Они допускают $13=6+4+3$ нумераций ребер. Из них 4 нумерации соответствуют рациональным функциям с тремя полюсами $(K=3, \chi=2)$, а 9 - эллиптическим функциям $(K=1, \chi=0)$.

\section{§11. Приложения в теории экстатических точек плоских кривых}

Речь идет о точках, в окрестности которых плоская кривая аппроксимируется алгебраическими кривьми степени $n$ с бо́льшим порядком касания, чем обычно.

ОПРЕДЕЛЕНИЕ. Точкой $n$-nерегиба гладкой плоской кривой назьвается точка, в которой кратность пересечения кривой с подходящей алгебраической кривой степени не выше $n$ необычно велика, а именно превосходит число

$$
d(n)=\frac{n(n+3)}{2}
$$

ЗАмЕЧАНИЕ. Число $d(n)$ - это размерность пространства плоских алгебраических кривых степени не вьше $n$. В каждой точке гладкой кривой сушествует алгебраическая кривая степени не выше $n$, пересекаюшая данную кривую с кратностью не ниже $d(n)$.

ПримеР 1. $d(1)=2$. Точки 1-перегиба - это обычные точки перегиба (нули кривизны).

ПримеР 2. $d(2)=5$. Точки 2 -перегиба-это точки, в окрестности которых кривая аппроксимируется коникой с погрешностью, не превосходящей $C t^{6}$ (где $t$ - расстояние от точки).

Точки 2-перегиба называются поэтому секстатическими.

Д. Эйзенбуд предложил назьвать все точки $n$-перегибов кривой әкстатическими, так как они являются необычно привлекательными для алгебраического геометра, изучающего кривую.

Классическим обобшением теоремы о четырех вершинах является следующая

ТЕОРема (ср. [64]). Плоская выпуклая кривая имеет не менее шести секстатических точек $(6=d(2)+1)$.

Развитая вьше теория позволяет исследовать вопрос о числе точек $n$-перегиба для плоских кривых, получающихся из алгебраических кривых фиксированной степени 
мальми возмушениями. Например, в эту схему укладывается теорема Мёбиуса о трех точках перегиба малых возмущений проективной прямой $(3=d(1)+1)$.

Результаты, однако, получаются несколько неожиданньми (см. [30]):

ТЕОРема 1. Плоская кривая, достаточно близкая $к$ окружности, имеет не менее восъми точек 3-перегиба. Существуют возмущения, имеющие ровно восемь невырожсденных точек 3-перегиба.

ТЕОРема 2. Плоская кривая, достаточно близкая $к$ выпуклому овалу әллиптической кривой $y^{2}=p_{3}(x)$, имеет не менее десяти точек 3-перегиба. Существуют возмущения, имеющие ровно десять невырожденных точек 3-перегиба $(10=d(3)+1)$.

ДокаЗАТЕЛЬСТво ТЕОРемы 2. Рассмотрим отображсение Веронезе $V_{3}: \mathbb{R}^{2} \rightarrow$ $\mathbb{R}^{9}$, сопоставляющее точке $(x, y)$ точку, координатами которой являются значения всех девяти одночленов степени от 1 до $3,\left(x, y, x^{2}, x y, \ldots, y^{3}\right)$. Пусть $C$ - эллиптическая кривая, $C_{0}$ - ее выпуклый овал. Кривая $V_{3} C$ лежит в (аффиинной) гиперплоскости $\mathbb{R}^{8}$ в $\mathbb{R}^{9}$, заданной уравнением кубической кривой $C$.

ЛЕмма. Кривая $V_{3} C_{0}$ вылиукла.

ДокаЗАТЕльство. Пересечение кривой $V_{3} C_{0}$ с гиперплоскостью $H$ в $\mathbb{R}^{8}$ состоит из образов точек пересечения овала $C_{0}$ с кубической кривой $C^{\prime}$, соответствуюшей содержашему $H$ 8-мерному аффинному пространству в $\mathbb{R}^{9}$.

По теореме Безу кубические кривые $C$ и $C^{\prime}$ пересекаются не более, чем в девяти точках. Но на овале $C_{0}$ лежит четное число точек пересечения. Значит, их число не превосходит восьми, что и доказывает выпуклость кривой $V_{3} C_{0}$. Лемма доказана.

Рассмотрим теперь малое (с производными) возмущение овала. Образ возмущенной кривой при отображении Веронезе - кривая в $\mathbb{R}^{9}$, близкая к выпуклой кривой в $\mathbb{R}^{8}$. По теореме о точках уплощения этот образ имеет не менее десяти точек уплощения, где кривая необычно близка к своей соприкасающейся гиперплоскости. Эти точки и являются образами точек 3 -перегиба при отображении Веронезе $V_{3}$ (соприкасающаяся гиперплоскость как раз и определяет искомую аппроксимирующую кубическую кривую).

Пример возмущения с ровно десятью точками 3-перегиба построен в [30].

ЗАмЕчАниЕ. Вопрос о числе точек $n$-перегиба для $n>3$ совершенно не исследован (даже для кривых, близких к прямой, к окружности или к алгебраической кривой степени $n)$.

\section{§12. Многомерные обобщения теории Штурма}

Хорошо известно, что прямолинейные попытки перенести теоремы о нулях линейных комбинаций собственных функций на многомерный случай оказываются неудачными (см., например, “теорему Германа" в [65]). Геометрические приложения теоремы Гурвица типа Штурма, описанные вьше, подсказывают совершенно другие обобщения, приводящие, в частности, к гипотезам о сферических функциях, формулируемьм ниже. 
Начнем с многомерного аналога теоремы Мёбиуса о трех точках перегиба возмущения проективной прямой на проективной плоскости. Мы должны определить многомерньй аналог точки перегиба плоской кривой.

Рассмотрим проективную плоскость $\mathbb{R} P^{2}$ в проективном пространстве $\mathbb{R} \mathrm{P}^{3}$. Все точки плоскости являются параболическими: вторая квадратичная форма в них вырождена (и даже равна нулю). Заметим, что понятие параболической точки на поверхности в проективном пространстве - проективное: речь идет о характере касания плоскости и поверхности в точке. Параболические точки поверхности общего положения образуют гладкую кривую (рис. 15). Эта кривая делит поверхность на гиперболическую область (где имеется два вещественных асимптотических направления) и эллиптическую (где они комплексно-сопряжены). В параболических точках оба асимптотических направления совпадают. Параболическая линия поверхности общего положения оснащена гладким полем асимптотических направлений. В отдельных точках это асимптотическое направление вдобавок касается параболической кривой.

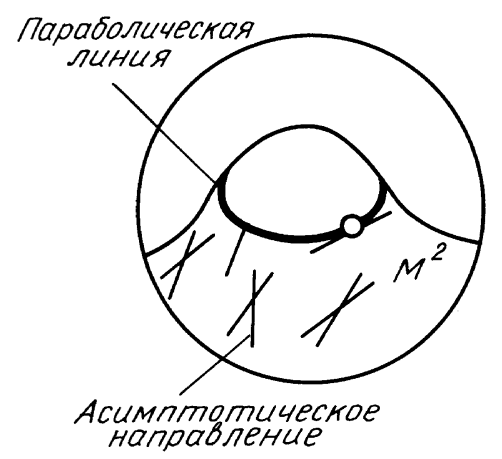

Р ис. 15. Параболическая линия и асимптотические направления на поверхности

На двойственной поверхности, лежащей в проективно двойственном проективном пространстве, параболическая линия исходной поверхности обшего положения соответствует ребру возврата. Это ребро возврата полукубического типа само имеет особые точки (точки возврата также полукубического типа). Они соответствуют точкам касания асимптотического направления исходной поверхности с ее параболической линией. Особенность двойственной поверхности в такой точке - обычньй ласточкин хвост (рис. 16).

Аналогом точки перегиба плоской кривой является параболическая кривая поверхности в проективном пространстве, снабженная точками касания асимптотического направления с этой кривой. На двойственной поверхности им соответствуют ребра возврата и ласточкины хвосты.

При попытке перенести теорему Мёбиуса о трех точках перегиба на эту ситуацию возникает вопрос: какое минимальное число ребер возврата и какое минимальное число ласточкиных хвостов имеет двойственная поверхность маловозмущенной проективной плоскости в $\mathbb{R} \mathrm{P}^{3}$ ?

Этот вопрос не решен даже для инфинитезимальных возмушений плоскости. 


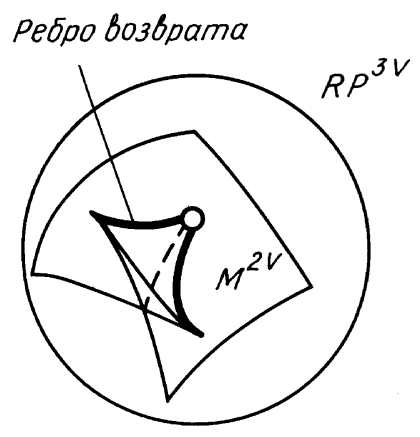

Р ис. 16. Ласточкин хвост на ребре возврата двойственной поверхности

Естественно начать с алгебраических возмущений минимальной возможной степени, т.е. с кубических поверхностей, близких к плоскости. Вопрос о числе ребер возврата и ласточкиных хвостов в этом случае решен Б. Сегре [66].

ТЕОРемА. Число компонент параболической линии на вещественной кубической поверхности общего положсения, гомеоморфной $\mathbb{R P}^{2}$, не меньше четьрех, причем число ласточкиных хвостов на двойственной поверхности равно uecmu.

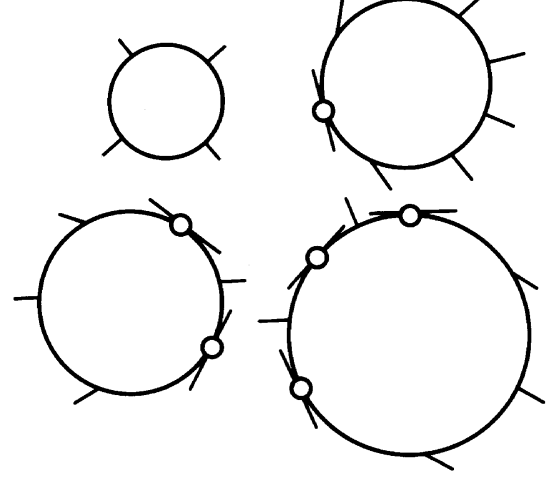

Рис. 17. Распределение особенностей по ветвям параболической кривой

ЗАмечАниЕ. Вычислительные эксперименты $\Phi$. Аикарди показали, что распределение шести ласточкиных хвостов по четырем ребрам возврата таково: $6=0+1+2+3$ (рис. 17). Е. Шустин, которому я сообшил об этом наблюдении, доказал (используя метод Сегре) неизбежность именно такого распределения точек касания асимптотических направлений с параболической кривой. При этом оказывается, что четыре “овала” параболической линии стягиваемы на $\mathbb{R P}{ }^{2}$ и ограничивают там непересекающиеся диски.

Таким образом, аналогом теоремы Мёбиуса явилась бы следующая 
ПЕРВАЯ ГИПОТЕЗА АИКАРДИ. Число связных компонент параболической линии малого ( с производньми) возмущения общего положения проективной плоскости в проективном пространстве не меньше четырех, а число точек возврата двойственной поверхности не меньше шести. При этом в минимальном случае, т.е. когда компонент четыре, а ласточкиных хвостов 6, они распределены по ребрам возврата так: $6=0+1+2+3$, а все четыре компоненты параболической линии ограничивают на поверхности непересекающиеся диски.

Возмушения проективной плоскости естественно описьвать при помоши рядов $\Phi$ рье по нечетным сферическим гармоникам.

ОПРЕДЕЛЕНИЕ. Функция на сфере в $\mathbb{R}^{m}$ (в частности, на окружности, если $m=2$ ) назьвается нечетной, если ее значения в противоположных точках сферы противоположны: $f(-x)=-f(x)$.

Теореме Мёбиуса о точках перегиба соответствует такой “нечетньй” вариант теоремы Гурвица типа Штурма:

ТЕОРемА. Нечетная вещественная функиия на окружности, ортогональная синусу и косинусу аргумента, имеет на окружсности не менее иести нулей.

ДокАЗАТЕльство. В ряд Фурье нечетной функции входят лишш нечетные гармоники. Поэтому первая из гармоник, входящих в ряд с ненулевым коэффищиентом третья или более высокая. Теорема Гурвища доставляет требуемые шесть нулей.

ЗАмЕчАниЕ. Если нечетная вещественная функция на окружности ортогональна первьм $2 n$ нечетным гармоникам

$$
\sin t, \cos t, \ldots, \sin (2 n-1) t, \cos (2 n-1) t,
$$

то число нулей на окружности не менњше $4 n+2$ (а на проективной прямой $S^{1} / \pm 1-$ не менњше, чем $2 n+1)$.

Теперь обратимся к многомерному случаю.

Пусть $f: S^{m-1} \rightarrow \mathbb{R}$ - нечетная функция на единичной сфере в евклидовом пространстве $\mathbb{R}^{m}$. Мы сопоставим этой функции волновой фронт (который на самом деле есть не что иное, как поверхность, двойственная определенному функцией $f$ малому возмушению проективной гиперплоскости $\mathbb{R} \mathrm{P}^{m-1}$ в $\mathbb{R} \mathrm{P}^{m}$ : смысл функции $f$ - это нормальная вариация проективной гиперплоскости).

ОПРЕДЕЛЕНИЕ. Волновым фронтом функиии $f$ назьвается образ единичной сферы $S^{m-1}$ при отображении $\operatorname{grad} F$, где $F$ - однородная функция степени 1 в $\mathbb{R}^{m}$, совпадающая с $f$ на сфере радиуса 1 .

ЗАмЕчАниЕ 1 . Функция $F$ удовлетворяет условию однородности степени 1 $F(c x)=c F(x)$ при всех вешественных $c$ (не обязательно положительных), так как функция $f$ нечетна.

ЗАмечАниЕ 2. Фронт является образом всего пространства $\mathbb{R}^{m} \backslash\{0\}$ при отображении $\operatorname{grad} F$, так как градиент однородной степени один функции постоянен вдоль выходящей из начала координат прямой. Фронт можно также рассматривать как образ не сферы, а проективного пространства $\mathbb{R} \mathrm{P}^{m-1}=S^{m-1} / \pm 1$ (так как значения градиента в противоположных точках сферы одинаковы). 
ПРИМЕР. При $m=2, f=\cos 3 t$ ( $t$ - полярный угол на окружности) фронтом оказывается гипоциклоида с тремя остриями. Она проходится целиком уже когда $t$ пробегает отрезок от 0 до $\pi$ (рис. 18 ).

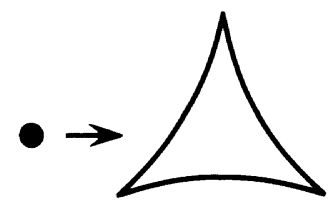

Рис. 18. Типичная деформация некоориентированного точечного волнового фронта на плоскости

ЗАмечАниЕ 3 . Разложим $f$ в ряд по сферическим гармоникам, $f=f_{1}+f_{3}+f_{5}+$ … Первый член ряда, $f_{1}$, соответствует линейной однородной функции $F_{1}$, градиент которой постоянен. Поэтому изменение $f_{1}$ приводит всего лишш к перемешению фронта в $\mathbb{R}^{m}$ как целого и не влияет на число и расположение его особенностей. Мы можем поэтому предполагать с самого начала, что ряд по сферическим гармоникам начинается с членов степени $3: f=f_{3}+f_{5}+\cdots$.

Если старшие гармоники отсутствуют, то вопрос сводится к теореме Б. Сегре:

Теорема. Волновой фронт в $\mathbb{R}^{3}$, соответствующий кубической гармонике $f_{3}$ (ограничению гармонического многочлена третьей степени общего положения от трех переменных на сферу $S^{2}$ ), имеет четыре ребра возврата и иесть ласточкиньих хвостов.

Вопрос о том, можно ли уменьшить число ребер возврата или особых точек, добавляя высшие гармоники, остается открытьм (даже если добавлять только гармоники степени пять).

Продолжая аналогию с теоремой Гурвица типа Штурма, естественно рассмотреть случай, когда третьи гармоники отсутствуют, так что ряд начинается прямо с пятых гармоник.

Численные эксперименты $\Phi$. Аикарди обнаружили волновые фронты, соответствуюшие сферической функции $f_{5}$ степени 5 , с (не менее чем) восемью ребрами возврата и четырнадшатью ласточкиными хвостами.

ВТОРАЯ ГИПОТЕЗА АИКАРДИ. Если нечетная вещественная функиия $f$ общего положения ортогональна всем кубическим гармоникам, то соответствующий ей волновой фронт в $\mathbb{R}^{3}$ имеет не менее, чем восемь ребер возврата и не менее, чем четырнадиать ласточкиных хвостов.

Смысл обеих гипотез состоит в әкономии сложности: топологическая сложность простейших алгебраических представителей изучаемых объектов предполагается минимальной по сравнению со всеми другими представителями.

Общее положение об экономии сложности подтверждается многочисленными примерами в разных областях математики. Например, “гипотеза Тома" (недавно доказанная Кронкаймером и Мровкой на основе работ Дональдсона и Виттена с Зейбергом) утверждает, что неособая алгебраическая кривая в $\mathbb{C P}^{2}$ имеет минимальньй род 
по сравнению со всеми гладкими поверхностями, реализующими тот же класс двумерных гомологий.

Кронхаймер и Мровка доказали также гипотезу Милнора о минимальности алгебраической реализации развязьвания узла. Рассмотрим локально неприводимую комплексную (скажем, алгебраическую) кривую $f(z, w)=0$ в $\mathbb{C}^{2}$ с особой точкой 0 .

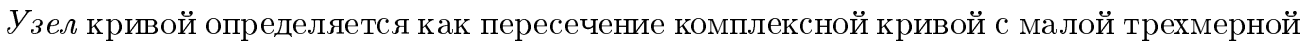
сферой $S^{3}$ в $\mathbb{C}^{2}$ с центром 0.

Гордиевым. числом узла назьвается минимальное число самопересечений, через которые надо пройти, чтобы развязать узел. Это - топологический инвариант узла.

Милнор определил чисто алгебраический инвариант особенности (считающий число двойных точек малого шевеления локального отображения $\mathbb{C} \rightarrow \mathbb{C}^{2}$, образом которого является кривая).

Оказывается, эта оценка точна, т.е. сложность развязываний не алгебраического происхождения может быть только бо́льшей, чем сложность, достигающаяся при алгебраической реализации развязывания.

Конечно, эти примеры подтверждения общего принципа экономии сложности при алгебраической реализации отнюдь не доказьвают ни его справедливость в сколько-нибудь обшей ситуации, ни справедливость сформулированных выше гипотез. Но для них, во всяком случае, не найдены опровергающие примеры.

ЗАмечАниЕ. Разобьем проективное пространство на большой шар и тонкую окрестность “бесконечно удаленной" проективной плоскости. Наша возмушенная поверхность разобьется на большой диск и тонкий лист Мёбиуса (вблизи бесконечности).

Зафиксируем какое-либо шевеление обшего положения нашей проективной плоскости в окрестности прямой на бесконечности и будем менять возмущение диска. Тогда первая гипотеза Аикарди преврашается в утверждение о параболических кривых и асимптотических направлениях на диске, вложенном в евклидово трехмерное пространство (с заданньми граничными условиями). Более того, этот возмущенньй диск можно считать графиком функции (если вся наша поверхность - возмушение плоскости).

Таким образом, первая гипотеза Аёкарди содержит множество гипотез типа принципа максимума для функций двух переменных (в зависимости от того, какое именно возмущение выбрано вблизи бесконечности).

Опровержение любого из этих следствий опровергло бы саму гипотезу.

Точно так же, зафиксировав возмущение диска, мы получаем гипотезы о вложениях листа Мёбиуса, которые можно сформулировать в терминах функций (или мёбиусовских функций) на окружности. Опровержение любой из получающихся (при разных выборах деформации диска) гипотез типа теории Штурма опровергло бы и исходную гипотезу.

Все приведенные вьше примеры показьвают, что в теории топологически необходимых особенностей волновых фронтов самые абстрактные математические конструкции переплетаются с компьютерным экспериментированием, а самые "чистые" области математики - с самыми "прикладньми" совершенно неразделимьм образом. 


\section{СПИСОК ЛИТЕРАТУРЫ}

[1] Weyl H. Invariants // Duke Math. J. 1939. V. 5. P. 500.

[2] Feynman R. Surely You're joking, Mr. Feynman! // Bantam Books. Toronto-New York, 1986. P. 191-198.

[3] Jacobi C. G. J. Vorlesungen über Dynamik. Berlin: G. Reiner, 1884.

[4] Zakalynkin V. M. Small deformations of Lagrangian collapse // Preprint: The University of Liverpool, 1995. P. 1-21.

[5] Arnold V.I. Sur les propriétés des projections Lagrangiennes en géométrie symplectique des caustiques // Cahiers de Mathématiques de la Decision. 1993. V. 9320. CEREMADE. P. 1-9.

[6] Табачников С. Л. Вокруг четырех вершин // УМН. 1990. Т. 45. №1. С. 229-230.

[7] Agrachev A. Methods of control theory in nonholonomic geometry // Proc. ICM Zürich: Birkhäuser, 1995. P. 1-11.

[8] Арнольд В.И. О топологических свойствах лежандровых проекций в контактной геометрии волновых фронтов // Алгебра и анализ. 1994. Т. 6. № 3. С. 1-16.

[9] Arnold V. I. Plane curves, their invariants, perestroikas and classifications // Singularities and Bifurcations / ed. V.I. Arnold. Providence: AMS, 1994. P. 33-91 (Adv. Sov. Math. V. 21).

[10] Arnold V. I. Topological invariants of plane curves and caustics // University Lecture Ser. V. 5. Providence: AMS, 1994. P. 1-60.

[11] Арнольд В. И. Инварианты и перестройки фронтов на плоскости // Труды МИАН. 1995. T. 209. C. $14-64$.

[12] Арнольд В.И. Геометрия сферических кривых и алгебра кватернионов // УМН. 1995. T. 50. № 1. C. 3-68.

[13] Aicardi F. Tree-like curves // Singularities and Bifurcations / ed. V. I. Arnold. Providence: AMS, 1994. P. 1-31 (Adv. Sov. Math. V. 21).

[14] Viro O. First degree invariants of generic curves on surfaces // Preprint U.U.D.M. Report 1994: 21. ISSN 1101-3591.: Department of Mathematics. Uppsala University, 1994. P. 1-22.

[15] Tabachnikov S. Invariants of smooth triple point free plane curves // Preprint U of A-R-77, 1994. P. 1-21.

[16] Шумакович А. Явные формулы для странности плоской кривой // Алгебра и анализ. 1995. T. 7; // S. Petersburg Math. J. 1995. V. 7.

[17] Polyak M. Invariants of plane curves and fronts via Gauss diagrams // Preprint. Max Planck Inst. für Math. Bonn, 1994. P. 1-13.

[18] Polyak M. On the Bennequin invariant of Legendrian curves and its quantisation // Preprint. Max Planck Inst. für Math. Bonn, 1995. P. 1-5; // C. R. Acad. Sci. Paris, 1995.

[19] Mikhalkin G., Polyak M. Whitney formula in higher dimensions // Preprint. Max Planck Inst. für Math. Bonn, 1995. P. 1-7.

[20] Aicardi F. Topological invariants of knots and framed knots in the solid torus // C. R. Acad. Sci. Paris. 1995. V. 321. P. 81-86.

[21] Aicardi F. Topological invariants of legendrian curves // C. R. Acad. Sci. Paris. 1995. V. 321. P. 199-204.

[22] Lin X.-S., Wang Z. Integral geometry of plane surves and knot invariants // J. Diff. Geom. 1995 (to appear).

[23] Ferrand E. On the Bennequin invariant and the geometry of wave fronts // Preprint. Ecole Polytechnique Palaiseau, 1995. P. 1-23.

[24] Arnold V. I. The Vassiliev theory of discriminants and knots // First Europ. Congr. Math. Paris, 1992; // Progress in Mathematics. V. 119. Basel: Birkhäuser, 1994. P. 1-29.

[25] Hurwitz A. Über die Fourieschen Konstanten integrierbarer Funktionen // Math. Ann. 1903. V. 57. P. 425-446.

[26] Kellogg O. The oscillation of functions on an orthogonal set // Amer. J. Math. 1916. V. 38. P. $1-5$. 
[27] Arnold V. I. On the number of flattening points of space curves // Preprint ISSN 1103 467X, 1SRN IML-R 1-94/95-SE, 1994. P. 1-13; // Sinai's Moscow seminar on Dynamical Systems, AMS Translations. Ser. 2. V. 171 (Adv. Sov. Math. 1995).

[28] Arnold V. I. Sur une propriété topologique des applications globalement canoniques de la mécanique classique // C. R. Acad. Sci. Paris. Sec. A. 1965. V. 261. P. 3719-3722.

[29] Ovsienko V., Tabachnikov S. Sturm Theory, Ghys theorem on zeroes of the Schwarzian derivative and flattening of Legendrian curves // Preprint CPT - CNRS. Luminy-Marseille, 1995. P. 1-13; // Selecta Mathematica, 1995 (to appear).

[30] Arnold V.I. Remarks on the extatic points of plane curves // Cahiers de Mathematiques de la Decision. V. 9529. CEREMADE (URA CNRS 749), 1995. P. 1-16; // Geometry and Singularity Theory / ed. V.I. Arnold, I. M. Gelfand, M. S. Smirnov. Basel: Birkhäuser, 1995.

[31] Арнольд В.И. Комментарий к "Об одной геометрической теореме" А. Пуанкаре // Пуанкаре А. Избранные сочинения. Т. 2. М.: Наука, 1972. С. 987-989.

[32] Conley C., Zehnder E. Birkhoff-Lewis fixed point theorem and a conjecture of V.I. Arnold // Inventiones Math. 1983. V. 73. P. 33-49.

[33] Chaperon M. Quelque questions de géométric symplectique [d'après, entre autres, Poincaré, Arnold, Conley et Zehnder] // Astérisque. 1983. V. 105-106. P. 231-249.

[34] Laudenbach F., Sikorav J.-C. Persistence d'intérsection avec la section nulle an course d'une: sotopic hamiltonienne daus le fibré cotangent // Inventiones Math. 1985. V. 82. №2. P. 349-358.

[35] Чеканов Ю. В. Лежандрова теория Морса // УМН. 1987. Т. 42. №4. С. 139-141.

[36] Floer A. Morse theory and Lagrangian intersections // J. Diff. Geom. 1988. V. 28. P. 513-547.

[37] Floer A. Proof of the Arnold conjecture and generalizations to certain Kahler manifolds // Duke Math. J. 1986. V. 53. P. 1-32.

[38] Floer A. Symplectic fixed points and holomorphic spheres // Comm. Math. Phys. 1989. V. 120. P. 576-611.

[39] Flor A., Hofer H. Symplectic homology. I // Math. Z. 1994. V. 215. P. 37-88.

[40] Gromov M. Pseudo-holomorphic curves in symplectic manifolds // Invent. Math. 1985. V. 82. P. $307-347$.

[41] Ono K. On the Arnold conjecture for weakly monotone symplectic manifolds // Preprint, 1994.

[42] Floer A. An instanton invariant for 3-manifolds // Comm. Math. Phys. 1988. V. 118. № 2. P. $215-240$.

[43] Atiyah M. New invariants of 3-and 4-manifolds // Sympos. Pure Math. AMS. Providence. 1988. V. 48. P. 285-289.

[44] Арнольд В.И. Первые шаги симплектической топологии // УМН. 1986. Т. 41. №6. C. $1-21$.

[45] Чеканов Ю. В. Критические точки квазифункций и производящие семейства лежандровых многообразий // Функц. анализ и его прилож. 1995. Т. 29 (в печати).

[46] Chaperon M. Familles génératrices des variétés Legendriennes // Floer Memorial Volume. Basel: Birkhäuser, 1995.

[47] Givental A. B. Nonlinear generalization of the Maslov index // Theory of singularities and its applications / ed. V.I. Arnold; // Adv. Sov. Math.. V. 1, 1990. P. 71-103.

[48] Kazarian M. Nonlinear version of Arnold's theorem on flattening points // C. R. Acad. Sci. Paris. 1996 (to appear).

[49] Shapiro B.Z., Shapiro M.Z. On the number of connected components in the space of closed nondegenerate curves on $S^{n} / /$ Bull. Amer. Math. Soc. 1991. V. 25. № 1. P. 75-80.

[50] Anisov S. Projective convex curves // Geometry and Singularity Theory / ed. V.I. Arnold, I. M. Gelfand, M. S. Smirnov. Basel: Boston, Birkhäuser, 1995.

[51] Арнольд В.И. Разветвленное накрытие $\mathbb{C P}^{2} \rightarrow S^{4}$, гиперболичность и проективная топология // Сиб. матем. журн. 1988. Т. 29. № 4. С. 36-47.

[52] Uribe R. On the vertices of convex space curves // C. R. Acad. Sci. Paris. 1995 (to appear). 
[53] Седых В. Д. Связь лагранжевых особенностей с лежандровыми при стереографической проекции // Матем. сб. 1994. Т. 185. № 12. С. 123-130.

[54] Овсиенко В., Хесин Б. Симплектические листы скобок Гельфанда-Дикого и гомотопические классы невырожденных кривых // Функц. анализ и его прилож. 1990. Т. 24. № 1. C. $62-69$.

[55] Arnold V. I. Bernoulli-Euler updown numbers associated with functions singularities, their combinatorics and arithmetics // Duke Math. J. 1991. V. 63. №2. P. 537-555.

[56] Арнольд В.И. Критические точки функций и классификация каустик // УМН. 1974. T. 29. № 2. C. $243-244$.

[57] Looijenga $\mathrm{E}$. The complement of the bifurcation variety of a simple singularity // Inventiones Math. 1974. V. 23. P. 105-116.

[58] Здравковска С. Топологическая классификация полиномиалњых отображений // УМН. 1970. Т. 25. № 4. С. 179-180.

[59] Thom R. L'equivalence d'une fonction differentiable et d'un polynome // Topology. 1965. V. 3. P. 297-307.

[60] Дэвис Ч. Свойства отображений некоторых чебышевских систем // ДАН СССР. 1958. T. 175. №2. C. 280-283.

[61] Davis C. Etrema of a polynomial // Am. Math. Monthly. 1957. V. 64. P. 679-680.

[62] Арнольд В.И., Варченко А. Н., Гусейн-Заде С. М. // Особенности дифференцируемых отображений. Т. 2. М.: Наука, 1984. С. 64-66.

[63] Глуцук А. Аналог теоремы Кэли для циклически-симметричных связных графов // УМH. 1993. T. 48. № 2. C. 233-234.

[64] Guieu L., Mourre E., Ovsienko V. Yu. Theorem on six vertices of a plane curve via Sturm theory // Preprint CPT, Luminy-Marseille, 1995.

[65] Гильберт Д., Курант Р. Методы математической физики. Т. 1, гл. 6. М.: Гостехиздат, 1933.

[66] Segre B. The non-singular cubic surfaces. Oxford: Clarendon Press, 1942.

[67] Gusein-Zade S. M., Natanson S. M. Arf invariant and Arnold invariant // Geometry and Singularity Theory / ed. V.I. Arnold, I.M. Gelfand, M.S. Smirnov. Basel: Boston, Birkhäuser, 1995.

[68] Горюнов В.В. Геометрия бифуркационных диаграмм простых проекций на прямую // Функц. анализ и его прилож. 1981. Т. 15. № 2. С. 77-82.

[69] Goryunov V. V. Subprincipal Springer cones and morsifications of Laurent polynomials and $D_{\mu}$ singularities // Singularities and Bifurcations / ред. V. I. Arnold. Providence: AMS, 1994. C. 163-188 (Adv. Sov. Math. V. 21).

[70] Арнольд В.И. Топологическая классификация комплексных тригонометрических многочленов и комбинаторика графов с одинаковым числом вершин и ребер // Функц. анализ и его прилож. 1996. Т. 30. № 1.

Математический институт

Поступила в редакцию

им. В. А. Стеклова РАН

04.09.1995

и Университет Париж-Дофин 